\title{
DISCLAIMER
}

This report was prepared as an account of work sponsored by an agency of the United States Government. Neither the United States Government nor any agency thereof, hor any of their employees, makes any wisranty, express or implied, or assumes any information, apparatus, product, or bility for the accuracy, completeness, or usefulness of any inforinge privately owned rights. Referprocess disclosed, or represents that its use would now or service by trade name, trademark, ence herein to any specific commercial product, process, or service by its endorsement, recommanufacturer, or otherwise does not necessarily constiment or any agency thereof. The views mendation, or favoring by the United States Governessarily state or reflect those of the

and opinions of authors expressed herein do states Government or any agency thereof.

\section{Transport of Gaseous C-14 from a Repository in Unsaturated Rock}

W. B. Light, P. L. Chambré, W. W.-L. Lee, and T. H. Pigford

Department of Nuclear Engineering

University of California

and

Earth Sciences Division

Lawrence Berkeley Laboratory

University of California

Berkeley, California 94720

September 1990

This work was supponted in part by the Director, Office of Civilian Radioactive Waste Management, Office of Systems integration and Regulations, Licensing and Compliance Division, of the U.S. Department of Energy under Contract No. DE-AC03-76SF00098. 


\begin{abstract}
We predict the transport of gaseous ${ }^{14} \mathrm{CO}_{2}$ from a nuclear waste repository in unsaturated rock using a porous-medium model. This model is justified if the appropriate modified Peclet number, which indicates equilibrium between gas in fractures and liquid in rock pores, is much less than unity. Numericaj illustrations are given which are applicable to the proposed repository at Yucca Mountain which is $\mathbf{3 5 0} \mathbf{m}$ underground. Maximum predicted concentrations of ${ }^{14} \mathrm{CO}_{2}$ near the ground surface are comparable to the USNRC limit for unrestricted areas. Maximum predicted dose rates above ground are less than one percent of background. Travel times are predicted to be hundreds to thousands of years. For some cases, we show that the release rate from the source has negligible effect on concentrations at the ground surface.
\end{abstract}




\section{TABLE OF CONTENTS}

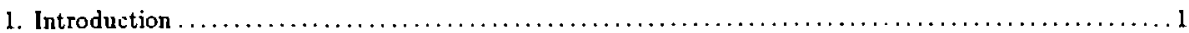

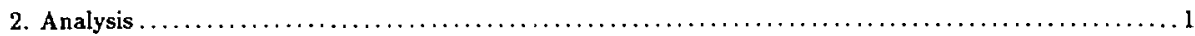

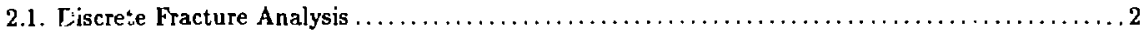

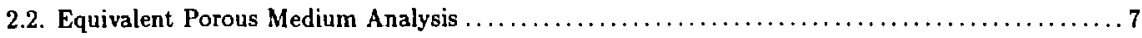

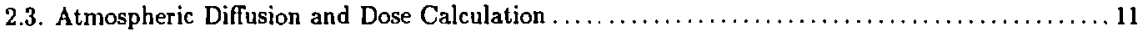

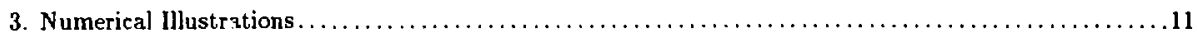

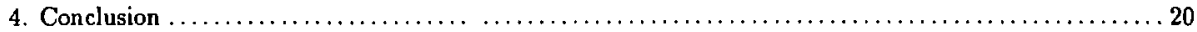

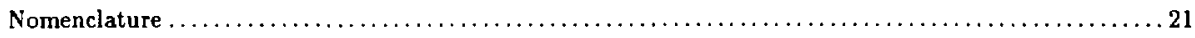

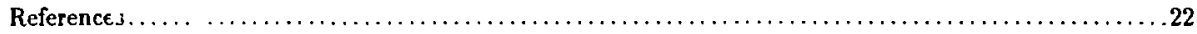

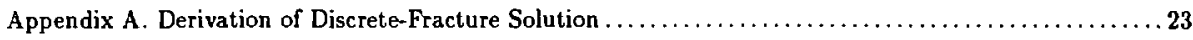

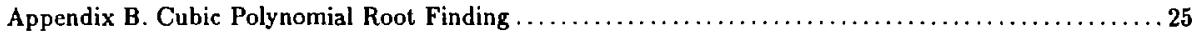

Appendix C. Derivation of Equivalent Porous Medium Solutions ......................... 27 


\section{INTRODUCTION}

Radioactive gases released from nuclear waste would have a direct pathway to the biosphere if such wastes were placed in unsaturated rock. This presents a new problem in assessing the potential health impacts of such releases and in complying witb regulations.

We analyze the transport of gaseous ${ }^{14} \mathrm{CO}_{2}$ in an unsaturated, fractured, porous medium with gas-phase advection and dispersion. Gases released into partially saturated, fractured rock tend to move in fractures, while vadose water is held inside the rock matrix. Strong convection flows are expected during the thermal phase of repository operation, carrying ${ }^{14} \mathrm{CO}_{2}$ toward the ground surface.

The region around the proposed repository at Yucca Mountain consists of a few alternating layers of welded and nonwelded tuff. The welded tuff layers are highly fractured while the nonwelded layers are sparsely fractured. Porosities are near 0.1 and liquid saturation is near 0.8 . Most of the permeability in the welded layers is due to fracture flow. Capillary action plays a large role in the fluid-flow system. Liquids tend to be bound by capillary force into the smaller pores, away from the fracture spaces, thus leaving the fractures open for gases to flow through. Other factors in the fluid-flow system are the recharge rate, heating from radioactive decay, and periodic changes (weather) at the ground surface.

In our analysis we first assess the interaction of ${ }^{14} \mathrm{CO}_{2}$ with vadose water as a possible retardation mechanism. Then we treat the combined fracture and pore matrix as an equivalent porous medium and predict ${ }^{14} \mathrm{C}$ concentrations, fluxes, travel times, and above-ground concentrations and dose rates.

This analysis differs from that proposed by Ross [1] in that we provide new support for the distributionequilibrium assumption by analyzing a discrete-fracture system; also, we provide complete analytic solutions to the equivalent porous medium problem with constant coefficients. We neglect calcite precipitation, thereby simplifying the equations given by Ross. Unlike Amter, Behl and Ross [2] and Knapp [3], who have also estimated ${ }^{14} \mathrm{C}$ travel-time, we include gas-phase dispersion and demonstrate its importance. We obtain essentially the same results as Knapp with a simpler formulation, but we do not see ${ }^{14} \mathrm{C}$ shock fronts. We use an atmospheric box-dispersion model after Schiager [4] to estimate above-ground concentrations and dose rates.

Parts of this work have been published previously [5]. This analysis has also been applied in a coupled analysis $\mathrm{Ci}^{14} \mathrm{C}$ leaked from nuclear waste containers $[6]$.

\section{ANALYSIS}

Our goal is to develop a model that can reliably estimate the release of ${ }^{14} \mathrm{C}$ to the accessible environment and the potential dose due to exposure above ground. We choose a simple mechanistic approach that will be reliable in extrapolating repository performance to the thousands of years required for the disposal of high level waste and will aid in understanding the qualitative behavior of the repository system.

The main force driving the transport of gaseous radionuclides to the biosphere is the convective flow of gas caused by radioactive-decay heat. Near the repository, this flow will be directed upward from the waste toward the ground surface. A steady-state gas flow model with constant, uniform upward gas flow will therefore capture the most important details for suitably short time windows.

We assume that ${ }^{14} \mathrm{C}$ is released from failed waste canisters as ${ }^{14} \mathrm{CO}_{2}(\mathrm{~g})$. Because $\mathrm{CO}_{2}$ dissolves readily in water, we expect transport of ${ }^{14} \mathrm{C}$ to be retarded by dissolution into vadose water. Some ${ }^{14} \mathrm{C}$ will react to form calcite and other minerals but the fraction in solid phases is difficult to predict and the amount is probably not significant compared to the fraction in gas and liquid phases. Thus we neglect precipitation into solid phases.

The degree to which $\mathrm{CO}_{2}$ dissolves in water is well known and the reaction rate is relatively fast. For ${ }^{14} \mathrm{CO}_{2}$, we adopt the equilibrium values for $\mathrm{CO}_{2}$ in pure water at infinite dilution. Corrections for the chemical difference between ${ }^{12} \mathrm{C}$ and ${ }^{14} \mathrm{C}$, capillary effects, and varying air and water compositions are small relative to other model uncertainties. The four aqueous species- $\mathrm{CO}_{2}$, carbonic acid, and bicarbonate and carbonate ions-account for almost all of the inorganic liquid-phase carbon. Using the equilibrium values for these species, a good approxintation can be made of the ratios of concentrations of ${ }^{14} \mathrm{C}(\mathrm{g})$ to ${ }^{14} \mathrm{C}(\mathrm{aq})$ in adjoining gas and liquid as a function only of temperature and $\mathrm{pH}$. 
We define the gas-liquid distribution coefficient

$$
K_{D}=\frac{\text { molar concentration of inorganic carbon in liquid }}{\text { molar concentration of inorganic carbon in gas }}
$$

Taking into account the four major aqueous species and $\mathrm{CO}_{2}$ in the gas phase

$$
K_{D}=\frac{\left[\mathrm{CO}_{2}(\mathrm{aq})\right]+\left[\mathrm{H}_{2} \mathrm{CO}_{3}(\mathrm{aq})\right]+\left[\mathrm{HCO}_{3}^{-}\right]+\left[\mathrm{CO}_{3}^{2-}\right]}{\left[\mathrm{CO}_{2}(\mathrm{~g})\right]}
$$

In terms of the gas constant $R$, the temperature $T$, the hydrogen ion concentration $\left[\mathrm{H}^{+}\right]$and the equilibrium constants $K_{\mathrm{H}}, K_{1}$ and $K_{2}$

$$
K_{D}=R T K_{\mathrm{H}}\left[1+\frac{K_{1}}{\left[\mathrm{H}^{+}\right]}+\frac{K_{1} K_{2}}{\left[\mathrm{II}^{+}\right]^{2}}\right]
$$

where

$$
K_{\mathrm{H}}=\frac{\left[\mathrm{CO}_{2}(\mathrm{aq})\right]+\left[\mathrm{H}_{2} \mathrm{CO}_{3}(\mathrm{aq})\right]}{p_{\mathrm{CO}_{2}}} \quad \mathrm{~K}_{1}=\frac{\left[\mathrm{H}^{+}\right]\left[\mathrm{HCO}_{3}^{-}\right]}{\left[\mathrm{CO}_{2}(\mathrm{aq})\right]+\left[\mathrm{H}_{2} \mathrm{CO}_{3}(\mathrm{aq})\right]} \quad \mathrm{K}_{2}=\frac{\left[\mathrm{H}^{+}\right]\left[\mathrm{CO}_{3}^{2-}\right]}{\left[\mathrm{HCO}_{3}^{-}\right]}
$$

and $p_{\mathrm{CO}}$ is the partial nressure of $\mathrm{CO}_{2}$ in atmosplieres.

Equilibrium data from Stumm and Morgan [7] and Phillips, Phillips and Skeen [8] were used to plot the curves in Fig. 1 showing $K_{D}$ as a function of $\mathrm{pH}$ for various fixed temperature values. Depending on whe $\mathrm{pH}$ and temperature, $K_{D}$ may be as low as 2 or as high as $\mathbf{4 0 0}$ for geologic conditions surrounding the repository. For numerical illustrations, we use $K_{D}=3$, which corresponds to $50^{\circ} \mathrm{C}$ and $\mathrm{pH} 7$.

\subsection{Discrete Fracture Analysis}

The main purpose of the fracture model is to investigate whether gas-phase carbon in the fractures is at equilibrium with liquid-phase carbon in the rock between the fractures. The tendency of the gas and liquid to equilibrate will be offset by the different flow velocities of the two phases and the physical separation between fractures and rock pores.

We consider a single vertical planar fracture filled with gas, adjacent to a fully saturated porous medium, as shown in Figure 2. Once we demonstrate local equilibrium using this model, we can infer local equilibrium for a partially saturated rock matrix.

We neglect dispersion in the fracture and assume that the gas is well-mixed over the width of the fracture so that the concentration is uniform in the fracture in the $y$-direction. We also assume uniformity in the $x$-direction for concentrations in the fracture and rock matrix, as well as a constant, upward gas velocity in the fracture. The governing equation for the fracture domain, incorporating the interaction with the adjacent porous medium, is then

$$
\frac{\partial C_{g}}{\partial t}+\lambda C_{g}+v_{g} \frac{\partial C_{g}}{\partial z}=\left.\frac{c D_{l}}{b} \frac{\partial C_{l}}{\partial y}\right|_{y=b} \quad z>0 \quad t>0
$$

where $C_{g}$ is the molar concentration of ${ }^{14} \mathrm{C}$ in gas, $C_{1}$ is the molar concentration in liquid, $v_{g}$ is the fracturegas velocity, $c$ is the rock-matrix porosity, $D_{l}$ is the pore-liquid diffusion coefficient, $b$ is the half width of the fracture ard $\lambda$ is the decay constant.

Within the porous matrix, the only transport mechanism considered is transverse diffusion; diffusion parallel to the fracture and advection are neglected. For a constant scalar diffusion coefficient, the mass balance in the rock matrix is

$$
\frac{\partial C_{l}}{\partial t}+\lambda C_{l}-D_{l} \frac{\partial^{2} C_{l}}{\partial y^{2}}=0 \quad y>b \quad z>0 \quad t>0
$$

The $z$-axis is defined to be in the upward vertical direction with the repository horizon at $z=0$. We prescribe at $z=0$ the gas-phase concentration of ${ }^{14} \mathrm{CO}_{2}$ in the fracture as a function of time representing a gaseous release from the waste containers,

$$
C_{g}(0, t)=\psi(t) \quad t>0
$$




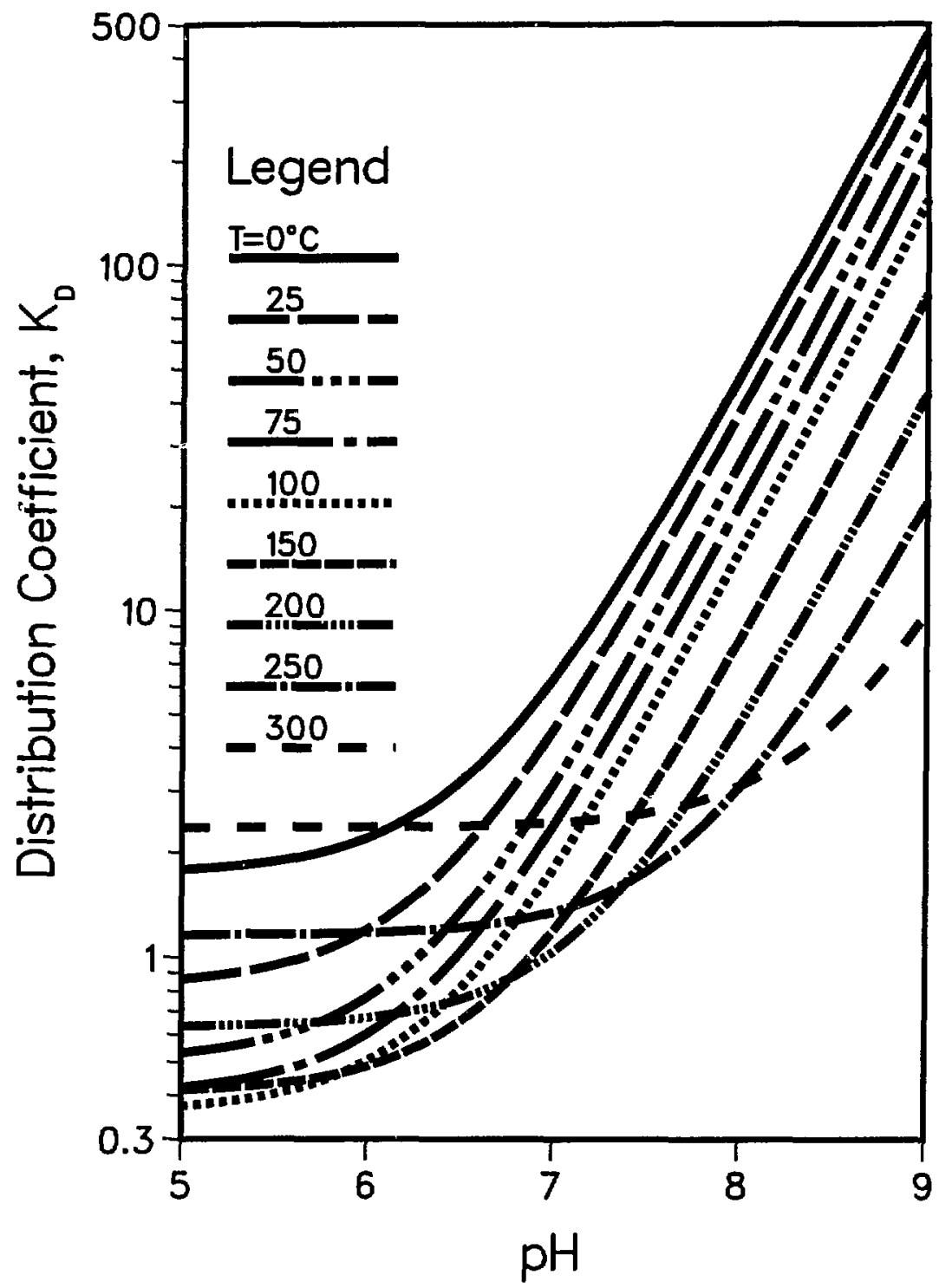

Figure 1. $K_{D}$ as a function of $\mathrm{pH}$ and temperature. 


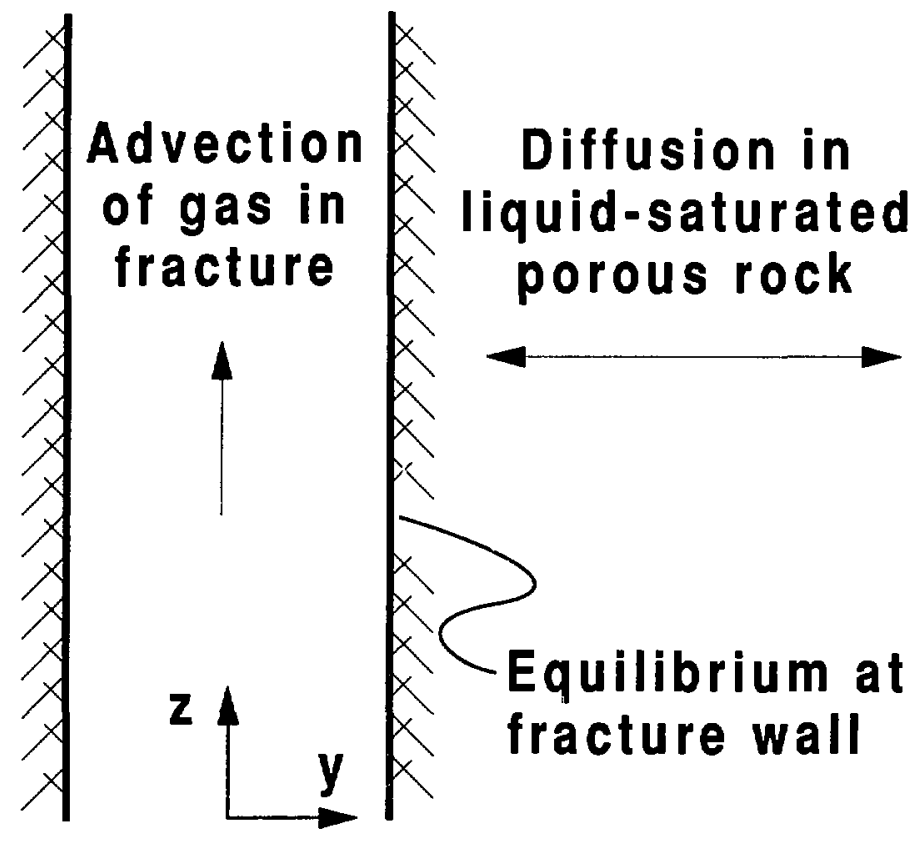

Figure 2. Fracture-model geometry showing a vertical planar fracture next to a porous rock matrix. 
At the fracture-wall interface where gas meets liquid, we assume the ratio between gas and liquid-phase concentrations to be giver by $K_{D}$,

$$
C_{l}(b, z, t)=K_{D} C_{s}(z, i) \quad z>0 \quad t>0
$$

The remaining initial and boundary conditions are

$$
\begin{aligned}
& C_{g}(z, 0)=0 \quad z>0 \\
& C_{7}(y, z, 0)=0 \quad y>b \quad z>0 \\
& C_{g}(\infty, t)=0 \quad t>0 \\
& C_{1}(\infty, z, t)=0 \quad z>0 \quad t>0
\end{aligned}
$$

The solution to this equation system is derived in Appendix $A$ and written here in terms of the dimensionless parameters $Y, Z, T$ and $\Lambda$. The concentration of ${ }^{14} \mathrm{CO}_{2}$ in the fracture is given by

$$
C_{g}(Z, T)=e^{-\Lambda z} h(T-Z) \int_{0}^{T-Z} \psi(T-Z-\tau) \frac{Z}{2 \sqrt{\pi \tau^{3}}} \exp \left(\frac{-Z^{2}}{4 \tau}-\Lambda \tau\right) d \tau \quad Z>0 \quad T>0
$$

and in the rock matrix

$$
C_{l}(Y, Z, T)=K_{D} e^{-\Lambda z} h(T-Z) \int_{0}^{T-Z} \psi(T-Z-\tau) \frac{Z+Y}{2 \sqrt{\pi \tau^{3}}} \exp \left[\frac{-(Z+Y)^{2}}{4 \tau}-\Lambda \tau\right] d \tau \quad Y, Z, T>0
$$

where

$$
Y=c K_{D} \frac{y-b}{b} \quad Z=\frac{z \epsilon^{2} K_{D}^{2} D_{l}}{v_{g} b^{2}} \quad T=\frac{t \epsilon^{2} K_{D}^{2} D_{l}}{b^{2}} \quad \Lambda=\frac{\lambda b^{2}}{\epsilon^{2} K_{D}^{2} D_{l}}
$$

At this point we choose to consider a delta-function impulse in time for the concentration of ${ }^{14} \mathrm{CO}_{2}(\mathrm{~g})$ flowing into the fracture at $z=0$. That is we prescribe $\psi(T)$ as follows

$$
\psi(T)=2 C \delta(T)
$$

where $C$ is a constant with units of concentration. We can express $C$ in terms of the amount released by integrating the release rate over time and equating that to the total amount released $J_{0}$. The release rate is equal to the cross-sectional area of the fracture, $A$, times the gas velocity, $v_{g}$, times the concentration, $\psi(T)$, leading to the equation

$$
\int_{0}^{\infty} A v_{g} \psi d t=I_{0}
$$

Recalling from the definition of $T$ that

$$
d t=\frac{b^{2}}{c^{2} K_{D}^{2} D_{1}} d T
$$

we can change the variable of integration and substitute $\psi(T)$ from (16) to get

$$
\left(\frac{2 A v_{g} C b^{2}}{\epsilon^{2} K_{D}^{2} D_{l}}\right) \int_{0}^{\infty} \delta(T) d T=I_{0}
$$

or

$$
C=\frac{I_{0} c^{2} K_{D}^{2} D_{l}}{A v_{g} b^{2}}
$$


The impulse-release form of solutions (13) and (14) is obtained by substituting $\psi(T)$ from (16) and integrating using the sifting property of the delta function to get the gas concentration in the fracture

$$
C_{g}(Z, T)=h(T-Z) \frac{C Z}{2 \sqrt{\pi(T-Z)^{3}}} \exp \left[\frac{-Z^{2}}{4(T-Z)}-\Lambda T\right] \quad Z>0 \quad T>0
$$

and the liquid-phase concentration in the matrix

$$
C_{l}(Y, Z, T)=h(T-Z) \frac{K_{\mathrm{D}} C(Z+Y)}{2 \sqrt{\pi(T-Z)^{3}}} \exp \left[\frac{-\left(Z+Y^{\prime}\right)^{2}}{4(T-Z)}-\Lambda T\right] \quad Y>0 \quad Z>0 \quad T>0
$$

A quantity of ${ }^{14} \mathrm{C}$ released as an impulse into the fracture will be advected upward in the fracture and will diffuse transversely in the matrix. The transverse diffusion causes the impulse to spread in both the $y$ and $z$ directions. The location of the peak of the concentration wave in the fracture, $Z_{\text {peak }}(T)$, is obtained by maximizing $C_{g}$ in (21) with respect to $Z$. This involves finding the proper root of the cubic polynomial

$$
Z^{3}-2(1+T) Z^{2}-2 T Z+4 T^{2}=0
$$

At early times both $T$ and $Z$ are very small. The asymptotic behavior of $Z_{\text {peak }}(T)$ at early times can be found by dropping the cubic terms from (23) and solving the resulting quadratic polynomial

$$
-2 Z^{2}-2 T Z+4 T^{2}=0
$$

The roots are $-2 T$ and $T$. The positive root gives the early-time asymptote

$$
\lim _{T \rightarrow 0} Z_{\text {peak }} \sim T
$$

In other words, the pulse, at early times, moves at a velocity $d Z / d T \sim 1$. Substituting the definitions of $Z$ and $T$ into this equation yields the real (dimensional) velocity $d z / d t \sim v_{g}$.

The solution to the original cubic equation is derived in Appendix B. The root corresponding to $Z_{\text {peak }}$ is

$$
Z_{\text {peak }}=\frac{2}{3}(T+1)+\frac{2}{3} \sqrt{4 T^{2}+14 T+4} \cos \left(\frac{1}{3} A \operatorname{rrcos}\left[\frac{8 T^{3}-12 T^{2}+42 T+8}{\left(4 T^{2}+14 T+4\right)^{3 / 2}}\right]+\frac{4 \pi}{3}\right)
$$

The asymptotic limit of (26) for large time is given by

$$
\lim _{T \rightarrow \infty} Z_{\text {peak }} \sim \sqrt{2 T}
$$
$T \gg 1$.

From this we see that the velocity of the concentration peak in the fracture decreases like $1 / \sqrt{t}$ for

Another interesting result, obtained from the solution for the concentration in the matrix, $C_{l}(Y, Z, T)$ in (22), is the location of the ${ }^{14} \mathrm{C}$ peak in the rock matrix, where $C_{l}$ is maximized with respect to $Y$ for given $Z$ and $T$ values. The result, denoted $Y_{\text {peak }}$, is given by

$$
Y_{\text {peak }}=\left\{\begin{aligned}
0, & T<Z+\frac{1}{2} Z^{2} \\
\sqrt{2(T-Z)}-Z, & T>Z+\frac{1}{2} Z^{2}
\end{aligned}\right.
$$

The asymptotic limit as $T$ goes to infinity is

$$
\lim _{T \rightarrow \infty} Y_{\text {peak }} \sim \sqrt{2} \bar{T}-Z
$$

From this equation we see that the wave peak develops into a straight line in the rock matrix region with slope $\partial Y_{\text {peak }} / \partial Z=-1$. The slope in the dimensional $y-z$ plane is given by $-1 / P$ where $P$ is the modified Peclet number defined

$$
P=\frac{b v_{g}}{\epsilon K_{D} D_{l}}
$$


When $P$ is much less than unity, the asymptote intersects the fracture at nearly right angles. Furthermore, the concentration along this asymptote is constant in the asymptotic limit $(T \gg 1)$ as determined by taking the limit of the ratio of peak concentration in the matrix $\left(C_{l}\right)$ to the peak concentration in the fracture $\left(C_{g}\right)$

$$
\lim _{T \rightarrow \infty} \frac{C_{l \text { peak }}}{C_{g \text { peak }}}=\lim _{T \rightarrow \infty} \frac{C_{l}\left(Y_{\text {peak }}, Z, T\right)}{C_{g}\left(Z_{\text {peak }}, T\right)}=K_{D}
$$

We expect the result to be a function of $Z$; however, we discover that for a fixed but large time the concentration along the wave crest in the matrix region is constant and equal to $K_{D}$ times the peak concentration in the gas phase in the fracture.

We can do a similar calculation for a partially saturated matrix instead of the fully saturated matrix above if we assume local equilibrium between gas and liquid-phase concentrations throughout the matrix. The asymptotic limits are then valid for $t \gg b^{2} /\left(\epsilon_{g}+\epsilon_{l} K_{D}\right)\left(\epsilon_{g} D_{g}+\epsilon_{l} K_{D} D_{l}\right)$ and the asymptotic wave-peak slope $\partial y_{\text {peak }} / \partial z$ is given by the negative inverse of the modified Peclet number redefined

$$
P=\frac{b v_{g}}{\epsilon_{g} D_{g}+\epsilon_{l} K_{D} D_{l}}
$$

The significance of this result is that when $P$ is very small $(P \ll 1)$ the asymptote in the matrix intersects the fracture at nearly right angles. This leads us to conclude that if $P$ is, in fact, much less than unity, we can assume that gas-phase concentrations of ${ }^{14} \mathrm{CO}_{2}$ in the fracture tend to be at equilibrium with concentrations in laterally adjoining rock matrix. For the real case with multiple fractures, we use this result to infer that concentrations in the fractures will be to a good approximation at equilibrium with concentrations in the rock matrix in directions transverse to the fractures.

\subsection{Equivalent Porous Medium Analysis}

The transport of a species in a porous medium with both gas and liquid phases may be described using the following two equations.

$$
\left(\frac{\partial}{\partial t}+\lambda\right) \epsilon_{g} C_{g}+\nabla \cdot\left(\mathbf{q}_{g} C_{g}\right)-\nabla \cdot\left(\epsilon_{g} \mathbf{D}_{g} \cdot \nabla C_{g}\right)+S_{g l}=f_{g}
$$

and

$$
\left(\frac{\partial}{\partial t}+\lambda\right) \epsilon_{l} C_{l}+\nabla \cdot\left(\mathbf{q}_{l} C_{l}\right)-\nabla \cdot\left(\epsilon_{l} \mathbf{D}_{l} \cdot \nabla C_{l}\right)-S_{g l}=f_{l}
$$

where $\lambda$ is the decay constant, $\epsilon_{g}$ is the gas-filled porosity, $\epsilon_{l}$ is the liquid-filled porosity, $C_{g}$ is the molar concentration of ${ }^{14} \mathrm{C}$ in the gas phase, $C_{l}$ is the mrlar concentration of ${ }^{14} \mathrm{C}$ in the liquid phase, $q_{y}$ is the gas Darcy velocity, $q_{l}$ is the liquid Darcy velocity, $\mathbf{D}_{g}$ is the dispersion coefficient in the gas phase, $\mathbf{D}_{l}$ is the dispersion coefficient in the liquid phase, $S_{g l}$ is the net reaction rate from gas phase to liquid, $f_{g}$ is the gas-phase source term and $f_{l}$ is the liquid-phase source term.

If there is equilibrium between ${ }^{14} \mathrm{C}$ in the fractures and in the rock-matrix pore liquid at a given $z$, we can treat the whole as an equivalent porous medium without regard to fracture details. Equations (33) and (34) can be added together to eliminate the gas-liquid reaction rate term $S_{g l}$ with the result

$$
\left(\frac{\partial}{\partial t}+\lambda\right)\left(\epsilon_{g} C_{g}+\epsilon_{l} C_{l}\right)+\nabla \cdot\left(\mathbf{q}_{g} C_{g}+\mathbf{q}_{l} C_{l}\right)-\nabla \cdot\left(\epsilon_{g} \mathbf{D}_{g} \cdot \nabla C_{g}+\epsilon_{l} \mathbf{D}_{l} \cdot \nabla C_{l}\right)=f
$$

where $f=f_{g}+f_{i}$ is the total source strength.

Assuming local equilibrium, we replace $C_{l}(\mathbf{r}, t)$ with $K_{D} C_{g}(\mathbf{r}, t)$ yielding

$$
\left(\frac{\partial}{\partial t}+\lambda\right)\left[\left(\epsilon_{g}+\epsilon_{l} K_{D}\right) C_{g}\right]+\nabla \cdot\left[\left(\mathbf{q}_{g}+\mathbf{q}_{l} K_{D}\right) C_{g}\right]-\nabla \cdot\left[\epsilon_{g} \mathbf{D}_{g} \cdot \nabla C_{g}+\epsilon_{l} \mathbf{D}_{l} \cdot \nabla\left(K_{D} C_{g}\right)\right]=f
$$

Generally the coefficients are functions of space and time. We assume here that they are constant and tiat the dispersion coefficients are scalar. We divide through by $\left(\epsilon_{g}+\epsilon_{l} K_{D}\right)$ to get 


$$
\left(\frac{\partial}{\partial t}+\lambda\right) C_{g}(\mathbf{r}, t)+\mathbf{v} \cdot \nabla C_{g}(\mathbf{r}, t)-D \nabla^{2} C_{g}(\mathbf{r}, t)=\frac{f(\mathbf{r}, t)}{\epsilon_{g}+\epsilon_{l} K_{D}}
$$

where

$$
\mathbf{v}=\frac{\mathbf{q}_{g}+\mathbf{q}_{l} K_{D}}{\epsilon_{g}+\epsilon_{l} K_{D}} \quad D=\frac{\epsilon_{g} D_{g}+\epsilon_{l} K_{D} D_{l}}{\epsilon_{g}+\epsilon_{l} K_{D}}
$$

Here $\mathbf{q}_{g}$ is the gas Darcy velocity and $\mathbf{q}_{/}$is the liquid Darcy velocity. The scalar diffusion coefficients $D_{g}$ and $D_{l}$ have been used in place of dispersion tensors.

Solutions to this equation are well known for various source terms, boundary conditions and initial conditions. We assume an infinite domain with the concentration equal to zero initially and vanishing at infinity for all time. This assumption does not allow for a boundary condition at the ground surface. In reality, the ${ }^{14} \mathrm{C}$ gas concentration at the ground surface is near zero because of diffusion into the atmosphere. Therefore, the ${ }^{14} \mathrm{C}$ flux may be greater than predicted here due to the increased concentration gradient at the ground surface.

We consider solutions to the problems of both a point source and an infinite plane source. For both of these geometries we consider release rates described by impulse, band and decaying-band functions of time. For a given release ate $\dot{M}(t)$, a point-source term can be written

$$
f(\mathbf{r}, t)=\delta(\mathbf{r}) \dot{M}(t)
$$

and an infinite-plar,e-source term can be written

$$
f(\mathbf{r}, t)=\frac{1}{A} \delta(z) \dot{M}(t)
$$

where $A$ is the area normal to $z$ over which the release rate $\dot{M}$ occurs. The band release rate is given by

$$
\dot{M}(t)=\frac{I_{J}}{t_{\mathrm{r}}}\left[h\left(t-t_{J}\right)-h\left(t-t_{J}-t_{\mathrm{r}}\right)\right]
$$

where $I_{J}=I_{e} e^{-\lambda t}$ is the releasable inventory at the failure time $t=t_{J}, I_{e}$ is the releasable inventory at the time of emplacement $t=0, h$ is the unit step function, $t_{J}$ is the time between emplacement and failure, and $t_{r}$ is the time between failure and the end of release. A. decaying band release is given by

$$
\dot{M}(t)=\frac{I_{J} e^{-\lambda\left(t-t_{\ell}\right)}}{i_{r}}-\left[h\left(t-t_{J}\right)-h\left(t-t_{J}-t_{r}\right)\right]
$$

The impulse release rate is obtained by taking the limit as $t_{r}$ goes to zero and can be written

$$
\dot{M}(t)=I_{f} \delta\left(t-\ell_{f}\right)
$$

The solution for the point-source case (39) is

$$
C_{g}(\mathbf{r}, t)=\int_{0}^{t} \frac{\dot{M}(\tau) \exp \left[-\frac{(r-v(t-r))^{2}}{4 D(t-r)}-\lambda(t-r)\right]}{\left(\epsilon_{g}+\epsilon_{1} K_{D}\right)[4 \pi D(t-\tau)]^{3 / 2}} d \tau \quad t>0
$$

Similarly, the solution for the infinite-plane-source case (10) is

$$
C_{g}(z, t)=\int_{0}^{t} \frac{\dot{M}(\tau) \exp [-\lambda(t-\tau)]}{\left(\epsilon_{g}+\epsilon_{1} K_{D}\right) A} G(z-v(t-\tau), t-\tau) d \tau, \quad t>0
$$

where

$$
G(z, t)=\frac{\exp \left[-z^{2} / 4 D t\right]}{\sqrt{4 \pi D^{t}}}
$$


For impulse releases, the point-source solution simplifies to

$$
C_{g}(x, y, z, t)=\frac{I_{f} e^{-\lambda\left(t-t_{f}\right)}}{\left(\epsilon_{g}+\epsilon_{l} K_{D}\right)\left[4 \pi D\left(t-t_{f}\right)\right]^{3 / 2}} \exp \left[\frac{-\left(x^{2}+y^{2}+\left[z-v\left(t-t_{f}\right)\right]^{2}\right)}{4 D\left(t-t_{f}\right)}\right]
$$

and the infinite-plane-source solution simplifies to

$$
C_{g}(z, t)=\frac{I_{f} e^{-\lambda\left(t-t_{f}\right)}}{\left(\epsilon_{g}+\epsilon_{l} K_{D}\right) A} G\left(z-v\left(t-t_{f}\right)_{1} t-t_{f}\right)
$$

From these solutions we are interested in obtaining additional information about the behavior of the system. In particular, we would like to know the maximum concentration at the ground surface, the flux at the ground surface, the maximum flux at the ground surface and the cumulative flux at the grounci surface. Our models deal with an infinite domain. We assume that the conditions predicted by our models approximate the real conditions between the rer.-citory horizon and the ground surface. To obtain the maximun values at the ground surface (or any other $z$ value), we take partial derivatives with respect to time and set them equal to zero. Here we restrict ourselves to the infinite-plane cases.

For the infinite-plane, impulse release case, the time at which the concentration peaks at a given distance $z$ from the source plane is determined by taking the partial derivative of (48) with respect to $t$, setting it equal to zero and solving for $t$. The result, denoted $t_{\text {cmax }}$, is

$$
t_{\mathrm{cmax}}=t_{f}+\frac{\sqrt{1+z^{2} v^{2} / D^{2}+4 z^{2} \lambda / D}-1}{v^{2} / D+4 \lambda}
$$

For the decaying-band case, consider first the solution form

$$
C_{g}(z, t)=\frac{I_{e} e^{-\lambda t}}{\left(\epsilon_{g}+\epsilon_{l} K_{D}\right) A t_{r}} \cdot \int_{M a r\left[n, t-t_{f}-t_{r}\right]}^{t-t_{l}} G(z-v \tau, \tau) d \tau, \quad t>t_{f}
$$

The integral in (50) can be expressed in terms of complementary erior functions as given by Berman et al. [9]

$$
\int_{a}^{b} G\left(z-v \tau, \tau, d \tau:=\frac{1}{2 v}[g(z, b)-g(z, a)]\right.
$$

where

$$
g(z, t)=\operatorname{erfc}\left(\frac{z-v t}{\sqrt{4 D t}}\right)-\exp \left(\frac{z v}{D}\right) \operatorname{erfc}\left(\frac{z+v t}{\sqrt{4 D !}}\right)
$$

An alternate form is

$$
g(z, t)=\operatorname{erfc}\left(\frac{z-v t}{\sqrt{4 D t}}\right)-\exp \left(\frac{-(z-v t)^{2}}{4 D t}\right) F\left(\frac{z+v t}{\sqrt{4 D t}}\right)
$$

where

$$
F(x)=e^{x^{2}} \operatorname{erfc}(x)
$$

The function $F(x)$ monotonically decreases from unity at $x=0$ to zero at $x=+\infty$ and can be accurately calculated by rational approximation [10]. This is especially useful for large $z$ calculations when the exponential term in (52) causes numerical overflow problems.

The solution (50) for a decaying band release from an infinite plane source can be written

$$
C_{g}(z, t)= \begin{cases}\frac{I_{e} e^{-\lambda t}}{2 v\left(\epsilon_{g}+\epsilon_{l} K_{D}\right) A t_{r}} g\left(z, t-t_{f}\right), & t_{f}<t \leq t_{f}+t_{r} \\ \frac{I_{e} e^{-\lambda t}}{2 v\left(\epsilon_{g}+\epsilon_{l} K_{D}\right) A t_{r}}\left[g\left(z, t-t_{f}\right)-g\left(z, t-t_{f}-t_{r}\right)\right], & t>t_{f}+t_{r}\end{cases}
$$


For this case, $t_{\mathrm{cmax}}$ is determined by solving $f(t)=0$ where $f(t)$ is given by

$$
f(t)= \begin{cases}{\left[\frac{-\lambda}{\partial v} g(z, \tau)+G(z-v \tau, \tau)\right]_{0}^{t-t_{j}},} & t_{f}<t \leq t_{f}+t_{r} \\ {\left[\frac{-\lambda}{2 v} g(z, \tau)+G(z-v \tau, \tau)\right]_{t_{-t_{f}-t_{r}},}^{t_{-}},} & t>t_{f}+t_{r}\end{cases}
$$

Knowing the concentration, we can calculate the lux as the sum of an advective term and a diffusive term

$$
\begin{aligned}
\phi(z, t) & =\left(q_{g}+q_{1} K_{D}\right) C_{g}(z, t)-\left(\epsilon_{g} D_{g}+\epsilon_{l} D_{l} K_{D}\right) \frac{\partial}{\partial z} C_{g}(z, t) \\
& =\left(\epsilon_{g}+\epsilon_{l} K_{D}\right)\left(v C_{g}(z, t)-D \frac{\partial}{\partial z} C_{g}(z, t)\right)
\end{aligned}
$$

For an impulse release from an infinite plane source, the expression simplifies to

$$
\begin{aligned}
\phi(z, t) & =\left(\epsilon_{g}+\epsilon_{l} K_{D}\right) \frac{z+v\left(t-t_{f}\right)}{2\left(t-t_{f}\right)} C_{g}(z, t) \\
& =\frac{I_{e} e^{-\lambda t}}{A} G\left(z-v\left(t-t_{\rho}\right), t-t_{f}\right)\left\{\frac{z+v\left(t-t_{f}\right)}{2\left(t-t_{f}\right)}\right\} \quad t>t_{f}
\end{aligned}
$$

The time at which the flux at a given location is maximum, $t_{\mathrm{pmax}}$, is given by a root of the cubic polynomial

$$
\left(v^{3}+4 v D \lambda\right)\left(t-t_{f}\right)^{3}+\left(z v^{2}+2 v D+4 z D \lambda\right)\left(t-t_{f}\right)^{2}+\left(6 z D-v z^{2}\right)\left(t-t_{f}\right)-z^{3}=u
$$

For a decaying-band release from an infinite plane source, the the expression simplifies to

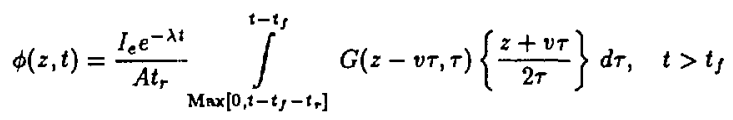

The integral in $(60)$ can be simplified by changing the integration variable to $p=(z-v \tau) / \sqrt{4 D \tau}$ with the result

$$
\int_{a}^{b} G(z-v \tau, \tau)\left\{\frac{z+v \tau}{2 \tau}\right\} d \tau=\frac{1}{2}\left[\operatorname{erfc}\left(\frac{z-v b}{\sqrt{4 D b}}\right)-\operatorname{erfc}\left(\frac{z-v a}{\sqrt{4 D a}}\right)\right]
$$

The solution (60) for a decaying band release from an infinite plane source is then

$$
\phi(z, t)= \begin{cases}\frac{I_{e} e^{-\lambda z}}{2 A t_{r}} \operatorname{erfc}\left(\frac{z-v\left(t-t_{f}\right)}{\sqrt{4 D\left(t-t_{f}\right)}}\right), & t_{f}<t \leq t_{\rho}+t_{r} \\ \frac{I_{e} e^{-\lambda t}}{2 A t_{r}}\left[\operatorname{erfc}\left(\frac{z-v\left(t-t_{f}\right)}{\sqrt{4 D\left(t-t_{f}\right)}}\right)-\operatorname{erfc}\left(\frac{z-v\left(t-t_{f}-t_{r}\right)}{\sqrt{4 D\left(t-t_{f}-t_{r}\right)}}\right)\right], & t>t_{\rho}+t_{r}\end{cases}
$$

For this case, the time at which the flux is at its peak is given by the root of $f(t)=0$ where $f(t)$ is given by

$$
f(t)= \begin{cases}{\left[\frac{-\lambda}{2} \operatorname{erfc}\left(\frac{z-v \tau}{\sqrt{4 D \tau}}\right)+G(z-v \tau, \tau)\left\{\frac{z+v \tau}{2 \tau}\right\}\right]_{0}^{t-t_{f}},} & t_{f}<t \leq t_{f}+t_{r} \\ {\left[\frac{-\lambda}{2} \operatorname{erfc}\left(\frac{z-v \tau}{\sqrt{4 D \tau}}\right)+G(z-v \tau, \tau)\left\{\frac{z+v \tau}{2 \tau}\right\}\right]_{t-t_{s}-t_{r}}^{t-t_{S}},} & t>t_{f}+t_{r}\end{cases}
$$




\subsection{Atmospheric Diffusion and Dose Calculation}

The above-ground ${ }^{14} \mathrm{CO}_{2}$ concentration $c$ is estimated by a method after Schiager [4] for estimating radon concentrations near uranium mill tailings piles

$$
c=\frac{\phi L}{\sigma_{z} U}
$$

where $L$ is the lateral extent of the repository, $U$ is the mean wind speed and $\sigma_{z}$ is the mean vertical mixing height. The vertical flux $\phi$ is evaluated at $350 \mathrm{~m}$ above an infinite plane source using the equivalent porous medium model. To convert above-ground ${ }^{14} \mathrm{CO}_{2}$ concentration to dose, we use the dose factor $10^{8}(\mathrm{mrem} / \mathrm{y}) /\left(\mu \mathrm{Ci} / \mathrm{cin}^{3}\right)$ from a recent NCRP report [11].

\section{NUMERICAL ILUSTRATIONS}

We illustrate the analytic results of the previous section using parameter values for a potential nuclear waste repository in unsaturated tuff at Yucca Mountain. The quick release of ${ }^{14} \mathrm{C}$ from waste containers is inost likely during the thermal period when waste temperatures are high. Also associated with the thermal period are large thermally-driven gas velocities. One estimate for the gas velocity in fractures due tw repository heating is $22 \mathrm{~m} / \mathrm{y}(0.04 \mathrm{~m} / \mathrm{y}$ Darcy velocity) [12]. We assume zero liquid velocity. Other parameters include $\epsilon_{g}=0.02, \epsilon_{l}=0.08, K_{D}=3, D_{g}=50 \mathrm{~m}^{2} / \mathrm{y}$, and $D_{l}=3 \times 10^{-3} \mathrm{~m}^{2} / \mathrm{y}$. The diffusion parameters are based on measured values for molecular diffusion in gas [13] and liquid continuums and inciude ten-fold reductions due to tortuosity. Hydrodynanic dispersion is neglected. The value for the fracture half-width, $10^{-5} \mathrm{~m}$, is characteristic of reported values [14]. The half-life of ${ }^{14} \mathrm{C}$ is 5730 years.

The modified Peclet number (32) is approximately $2 \times 10^{-4}$. This indicates that ${ }^{14} \mathrm{C}$ flowing in fractures will spread quichly into the rock matrix between fractures and thus be retarded in accordance with the local-equilibria assumptions used in the equivalent-porous-medium model. Retardation by the liquid phase is incorporated in the advective transport velocity $\mathbf{v}$ given by $(38)$. This yields the speed that a ${ }^{14} \mathrm{C}$ plume would travel independent of dispersion. For the given set of values, $v$ is about $0.015 \mathrm{~m} / \mathrm{y}$, suggesting a time of $2300 \mathrm{y}$ for ${ }^{14} \mathrm{C}$ to travel $350 \mathrm{~m}$ from the repository to ground surface. However, dispersion will cause the plume to spread and the leading edge will arrive at the ground surface more quickly.

The concentration and flux at the surface depend on the strength of the sourge. The total amount of ${ }^{14} \mathrm{C}$ is estimated to be $1.5 \mathrm{Ci} / \mathrm{Mg} \mathrm{U}$ fuel at the time of emplacement; $1 \%$ is assumed to be available for quick release [15]. Given $1.5 \mathrm{Mg}$ U fuel per canister, there will be $0.02 \mathrm{Ci}$ of the available ${ }^{14} \mathrm{C}$ inventory per failed canister. For a total of $70,000 \mathrm{Mg} U$ fuel in a filled repository, this corresponds to approximately $1,000 \mathrm{Ci}$ of ${ }^{14} \mathrm{C}$ available for quick release if all containers fail. If we assume $20 \%$ container failure, an inventory of $200 \mathrm{Ci}$ (about $40 \mathrm{~g}$ or 3 moles) could be available for quick release for the entire repository [15]. This estimate neglects source decay between emplacement and failure times, which will be unimportant during the thermal period.

Figure 3 shows the gas-phase concentration of ${ }^{14} \mathrm{C}$ as a function of distance above a single failed container as predicted by the point-source impulse solution for a release of $0.02 \mathrm{Ci}$. Note how the plume moves and spreads in time. At 1500 years, much of the ${ }^{14} \mathrm{C}$ has reached the surface $(350 \mathrm{~m})$, demonstrating the effect of dispersion on travel time. The maximum concentration in the plume decreases significantly as the plume spreads. This results almost entirely from gas-phase dispersion because little decay occurs in 1500 years. We conclude therefore that dispersion has an important effect on both the travel time and the concentration at the ground surface for this data set.

Figure 4 shows the gas-phase concentration above an infinite-plane source with an impulse source strength, characteristic of 20 percent container failures distributed over the entire repository. Only longitudinal dispersion is active in transport from the plane source in Figure 4, resulting in less dispersive attenuation than shown for the point-source release in Figure 3.

In Figure 5, the same infinite-plane source is used to show the gas-phase concentration $350 \mathrm{~m}$ above the source for three different values of the gas Darcy velocity: the reference value of $0.04 \mathrm{~m} / \mathrm{y}$, as well as 0.004 and $0.4 \mathrm{~m} / \mathrm{y}$. For $q_{g}=0.04 \mathrm{~m} / \mathrm{y}$, the leading edge reaches the ground surface in about 700 years. The concentration peaks in 2,000 years at $3 \times 10^{-7} \mu \mathrm{Ci} / \mathrm{cm}^{3}$, and the wave passes after about 6,000 years. With the slower velocity of $0.004 \mathrm{~m} / \mathrm{y}$, it takes only slightly longer for the leading edge to reach the ground 


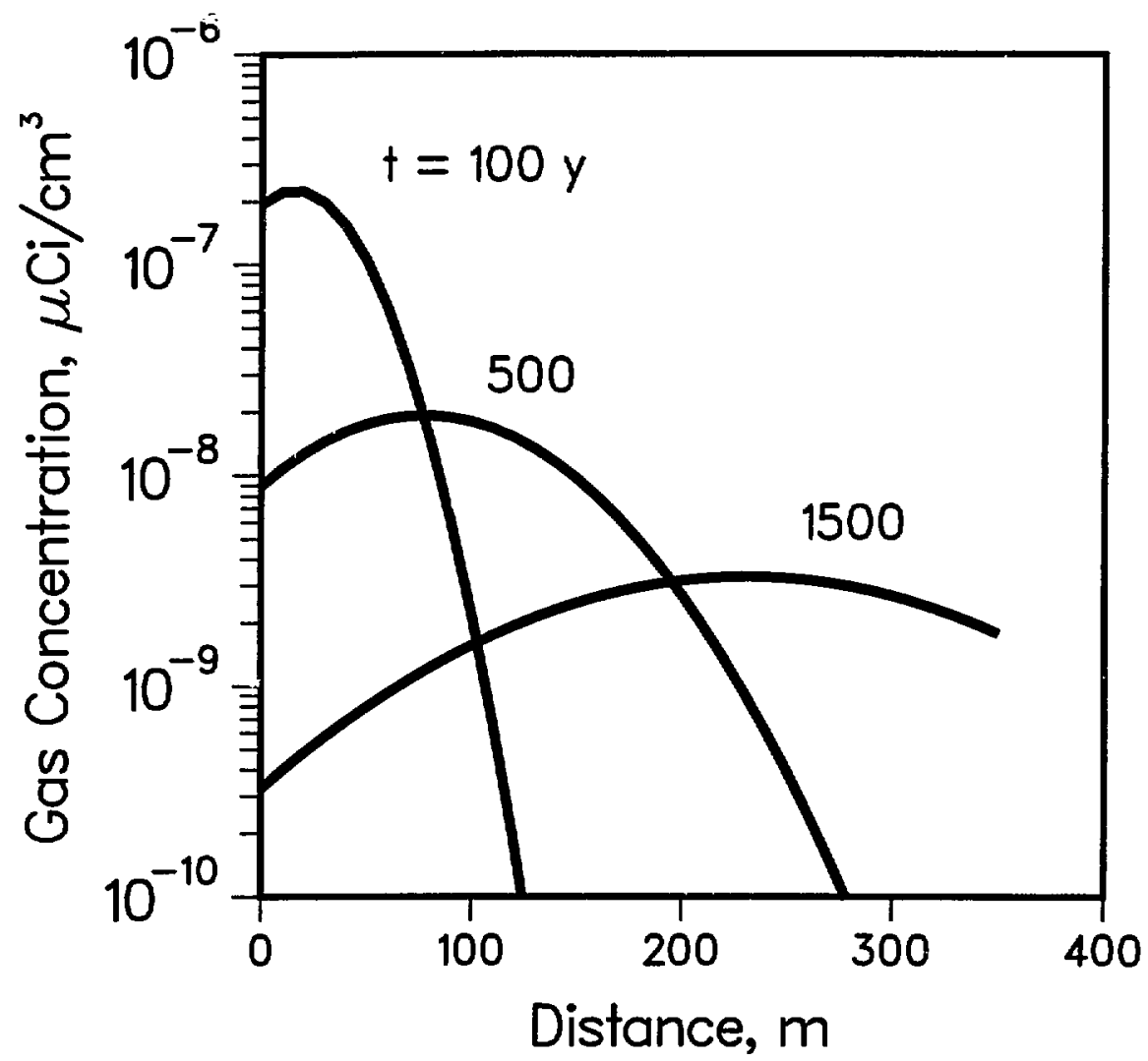

Figure 3. ${ }^{14} \mathrm{CO}_{2}$ concentration vs. distance for impulse release of $0.02 \mathrm{Ci}$ from a point source. Gas Darcy velocity is $0.04 \mathrm{~m} / \mathrm{y}$, release at time zero. 


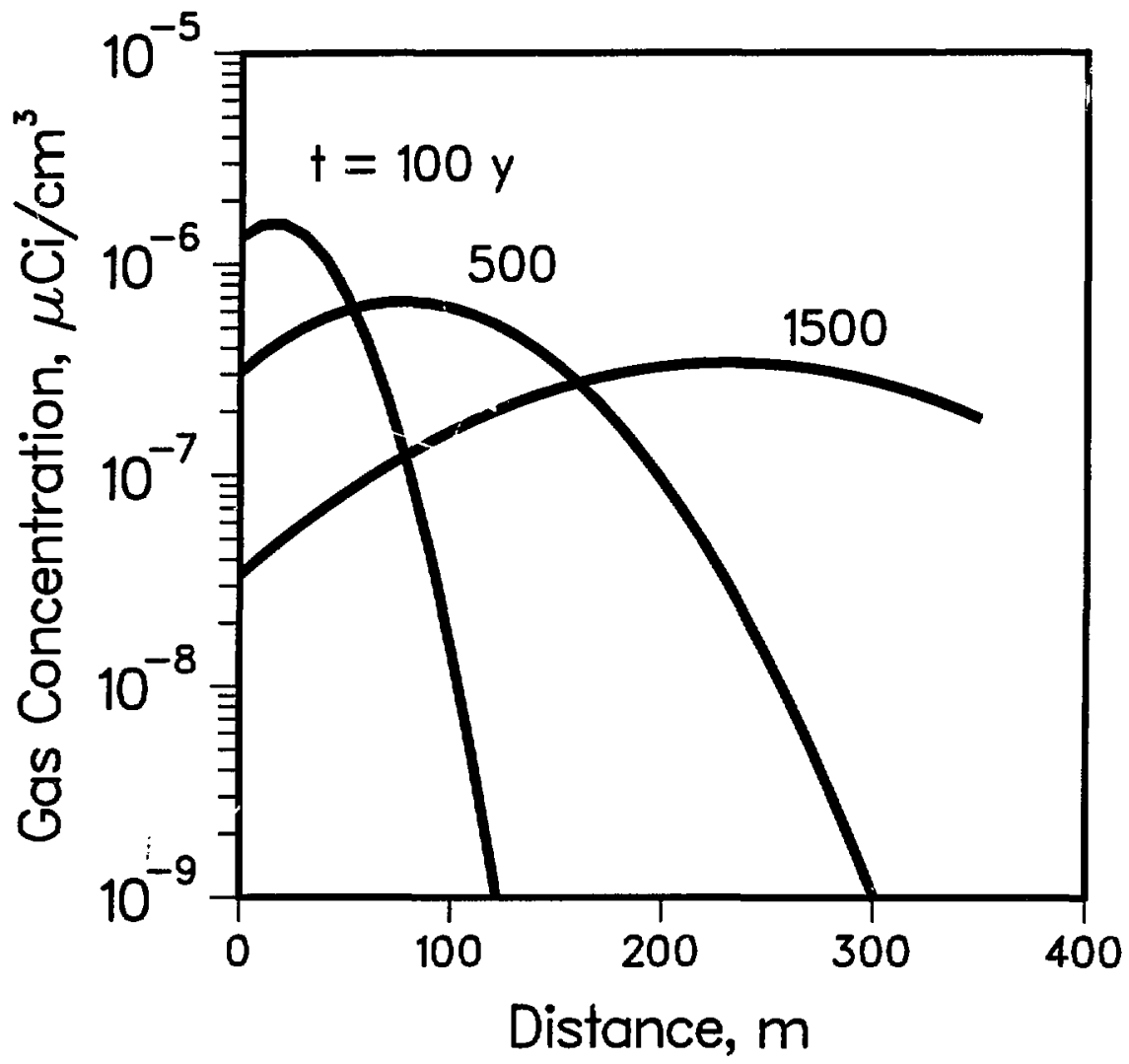

Figure 4. ${ }^{14} \mathrm{CO}_{2}$ concentration vs. distance for impulse release of $200 \mathrm{Ci}$ per $7 \times 10^{6} \mathrm{~m}^{2}$. Gas Darcy velocity is $0.04 \mathrm{~m} / \mathrm{y}$, release at time zero. 
surface, but the peak concentration is lower due to decay and dispersion. In addition, it takes much longer for the wave to pass through the ground. In contrast, when the velocity is $0.4 \mathrm{~m} / \mathrm{y}$, the wave passes through in 200-300 $y$ and the wave peak is much less attenuated by dispersion and decay, reaching $10^{-6} \mu \mathrm{Ci} / \mathrm{cm}^{3}$ at 230 years.

Figure 6 shows the gas-phase concentration 350 meters above an infinite-plane source due to a band release of $200 \mathrm{Ci}$ from a planar surface of $7 \times 10^{6} \mathrm{~m}^{2}$ for a duration of 1,000 years. This figure differs from Figure 5 in that the release is gradual; the $x$-axis shows elapsed time from the beginning of the release. Curves are plotted for the same three gas Darcy velocities as those used in Figure 5 . For $0.04 \mathrm{~m} / \mathrm{y}$, the leading edge reaches the ground surface after about 1,000 years. The concentration peaks at a little over $10^{-7} \mu \mathrm{Ci} / \mathrm{cm}^{3}$ and the wave passes after about 7,000 years. For both 0.04 and $0.004 \mathrm{~m} / \mathrm{y}$, the curves are only slightly different from the impulse-release curves in Figure 5, the latter being more dispersed and retarded. When the velocity is $0.4 \mathrm{~m} / \mathrm{y}$, the curve becomes flat on top because there is less time for dispersion. The wave takes less time to travel to the ground surface, approximately 230 years, and takes less time to pass through, about 230 years after the last releace irom the waste. At this fiow rate, dispersion becomes less important.

Figure 7 shows what happens to the peak concentration at the ground surface when the band-release duration is varied from 1 to 1,000 years while the total amount released is held constant at $200 \mathrm{Ci}$. For $q_{g}=0.04 \mathrm{~m} / \mathrm{y}$ and $0.004 \mathrm{~m} / \mathrm{y}$, the peak concentration is not affected by the band-release duration. At the higher gas Darcy velocity, $q_{g}=0.4 \mathrm{~m} / \mathrm{y}$, the peak concentration is decreased by the longer band-release duration. If the release duration is shorter than the travel time, as is true where the curves are flat, dispersion will attenuate the peak of the concentration wave as it travels and will tend to minimize differences caused by varying release durations. If the release duration is longer than the travel time, as is true where the $q_{s}=0.4 \mathrm{~m} / \mathrm{y}$ curve slopes down, dispersion does not attenuate the concentration and the smaller release rate of longer duration results in a lower peak concentration.

The cumulavive release at the ground surface through 10,000 years is shown in Figure 8 for the planesource, impulse release. All of the ${ }^{14} \mathrm{C}$ released from an impulse source is predicted to escape to the atmosphere in 300 years if $q_{g}=0.4 \mathrm{~m} / \mathrm{y}$. For the velocity $0.04 \mathrm{~m} / \mathrm{y}, 70$ percent will escape in 5,000 years, the rest having decayed. If the velocity is $0.004 \mathrm{~m} / \mathrm{y}$, about 10 percent will escape after 10,000 years and later.

To estimate above-ground ${ }^{14} \mathrm{CO}_{2}$ concentration, we choose $L=3000 \mathrm{~m}_{1} \sigma_{z}=20 \mathrm{~m}$ and $U=1 \mathrm{~m} / \mathrm{s}$ in (64). This is intended to produce a conservatively high estimate. The value for the vertical mixing height is consistent with atmospheric stability class $F$ (near stagnant) at a travel distance of $3000 \mathrm{~m}$ as extrapolated from Figure 9 in Schiager [4]. The flux at the ground surface is also needed. We have calculated the flux for impulse and decaying band releases using (58) and (62) for $K_{D}=3$ and $250, q_{g}=2.0,0.4,0.04$ and $0.004 \mathrm{~m} / \mathrm{y}$, and $t_{r}=0,300,1000,5000$ and 10,000 years. The amount released is based on an initial 1000-Ci releasable inventory. For the decaying band releases, the total amount released is less than $1000 \mathrm{Ci}$ due to decay.

The results from the flux calculations in terms of the maximum dose to an individual downwind oi the repository are summarized in Table 1 . There we see that the maximum dose predicted by this model is $10^{-2} \mathrm{mrem} / \mathrm{y}$ for an impulse release with $K_{D}=3$ anu $q_{g}=2.0 \mathrm{~m} / \mathrm{y}$. For $K_{D}=250$, the doses are much smaller.

Figure 9 shows the repository-average fractional release rate of ${ }^{14} \mathrm{C}$ at the ground surface based on the initial inventory. The right-hand scale shows the corresponding inhalation dose to an individual at the top of Yucca Mountain as a function of the release duration $t_{r}$ and the gas Darcy velocity $q_{g}$. The center curve, for a release duration of 1000 years and a calculated $q_{g}=0.04$ [12], is the most likely of the five cases in this figure. The peak dose is about $10^{-4} \mathrm{mrem} / \mathrm{y}$, or about $10^{-6}$ of background radiation dose. Increasing the release duration from 1000 to 10,000 years results in a peak dose about one order of magnitude lower. Note the sharp drop in dose following the end of the release period. For the release duration of 10,000 years, a lower average gas Darcy velocity is likely, hence we use $q_{g}=0.004 \mathrm{~m} / \mathrm{y}$ for the rightmost curve. Here the peak dose to an individual is about $2 \times 10^{-6} \mathrm{mrem} / \mathrm{y}$. The leftmost curve with the highest peak is for an instantaneous release with $q_{g}=0.4$. The peak dose is about $5 \times 10^{-3} \mathrm{mrem} / \mathrm{y}$, or $10^{-4}$ of background radiation. Figure 9 shows that for $q_{g} \leq 0.4$ and $t_{r} \geq 1000$, the repository-average fractional release rate at the ground surface is equal to or less than $10^{-5}$ per year, the USNRC release rate criterion for the engineered barrier system. 


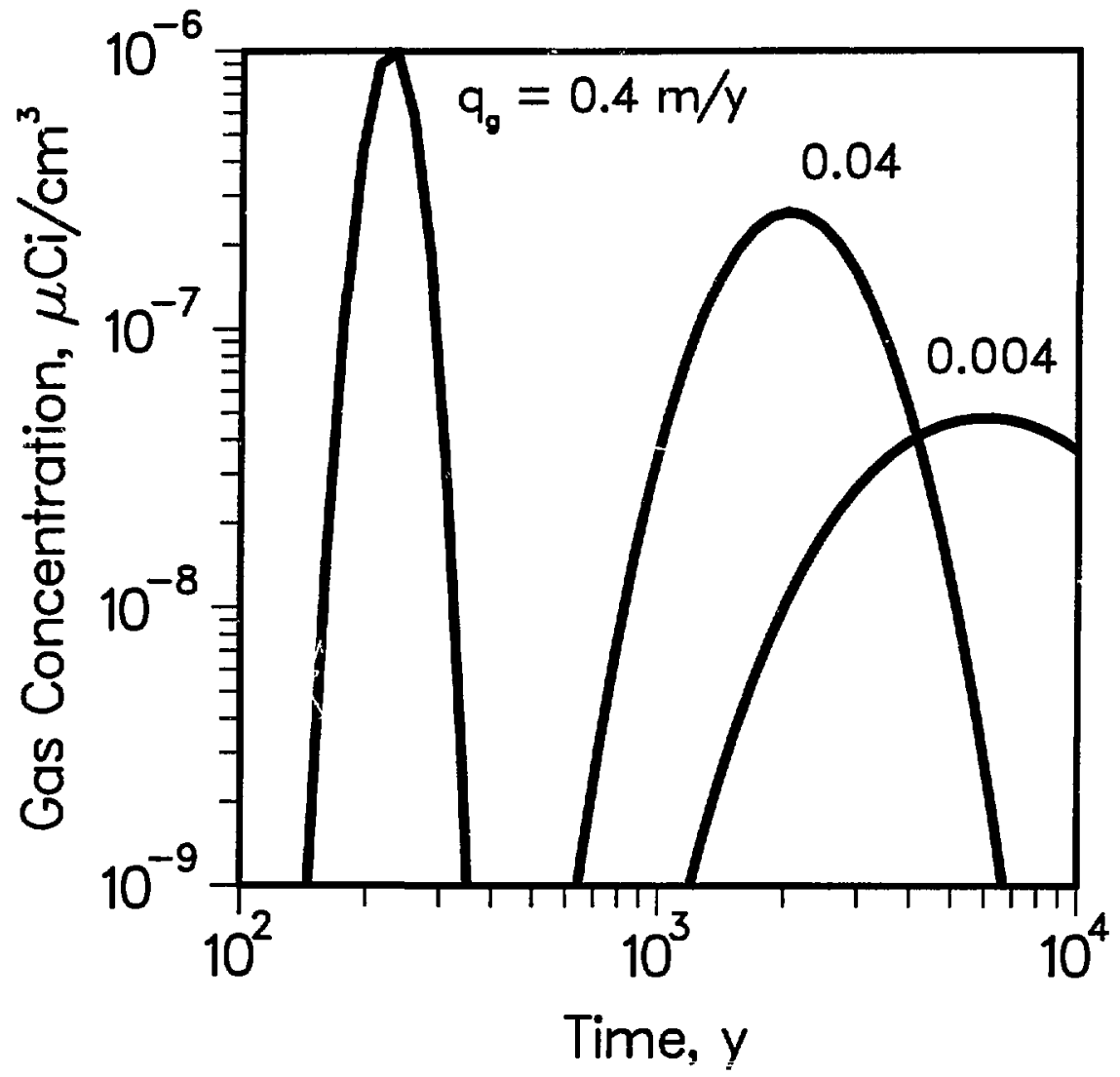

Figure 5. ${ }^{14} \mathrm{CO}_{2}$ concentration $350 \mathrm{~m}$ above an infinite plane source releasing $200 \mathrm{Ci}$ per $7 \times 10^{6} \mathrm{~m}^{2}$ in an impulse at time zero. 


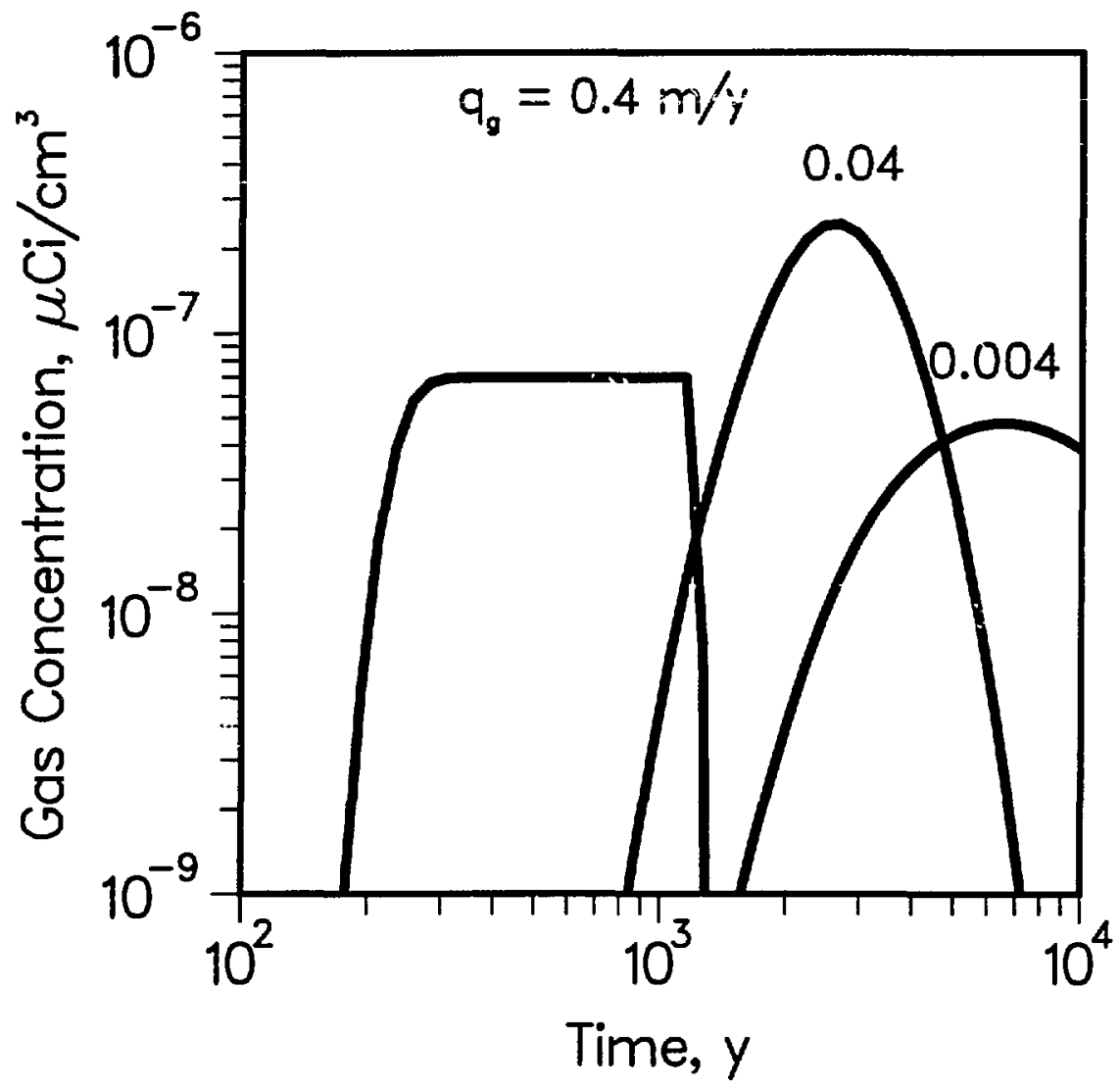

Figure 6. ${ }^{14} \mathrm{CO}_{2}$ concentration $350 \mathrm{~m}$ above an infinite plane source releasing $200 \mathrm{Ci}$ per $7 \times 10^{6} \mathrm{~m}^{2}$ over 1000 years, release starting at time zero. 


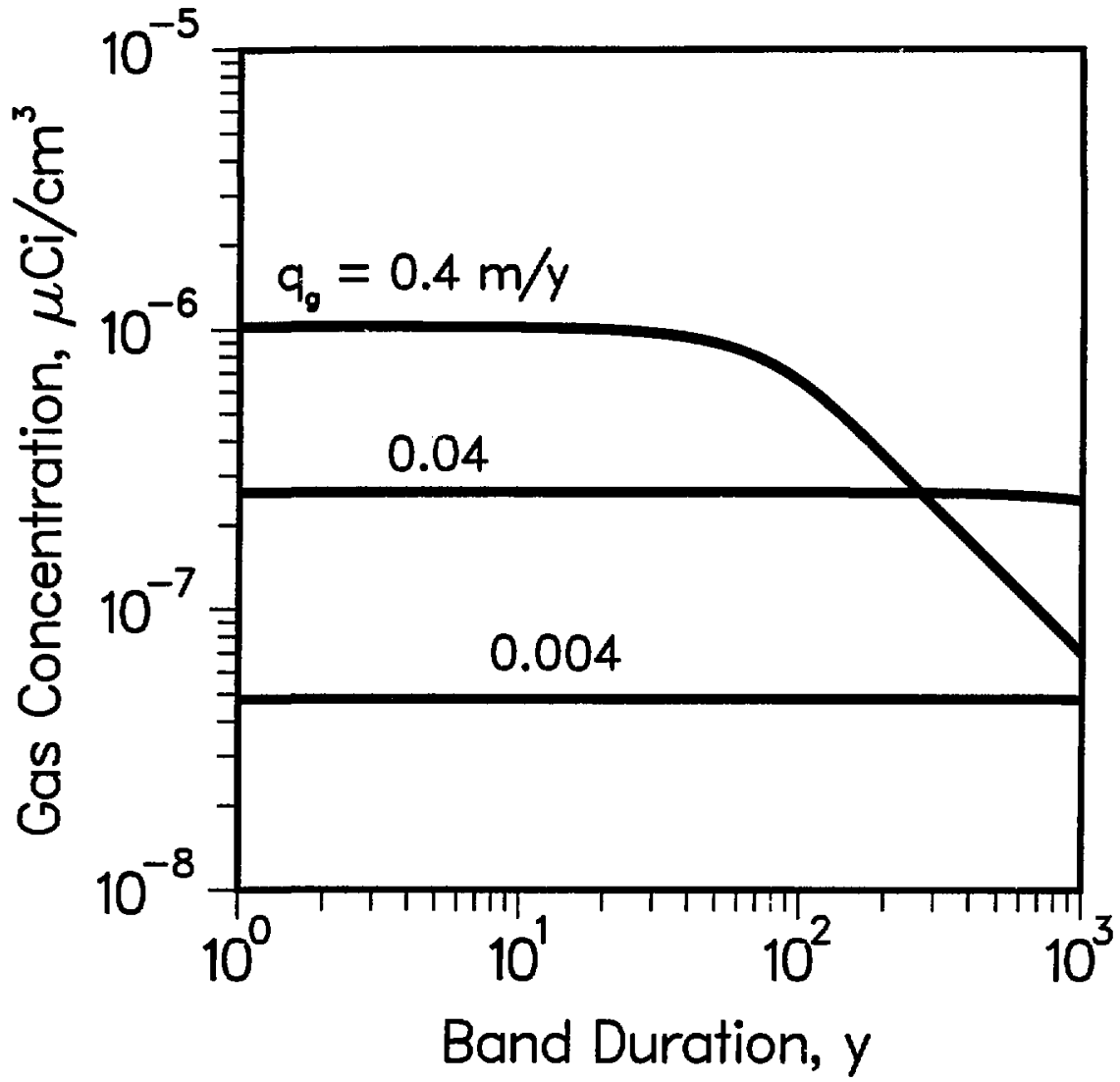

Flgure 7. Peak ${ }^{14} \mathrm{CO}_{2}$ concentration $350 \mathrm{~m}$ above infinite plane source as a function of the band release duration for $200 \mathrm{Ci}$ released per $7 \times 10^{6} \mathrm{~m}^{2}$. 


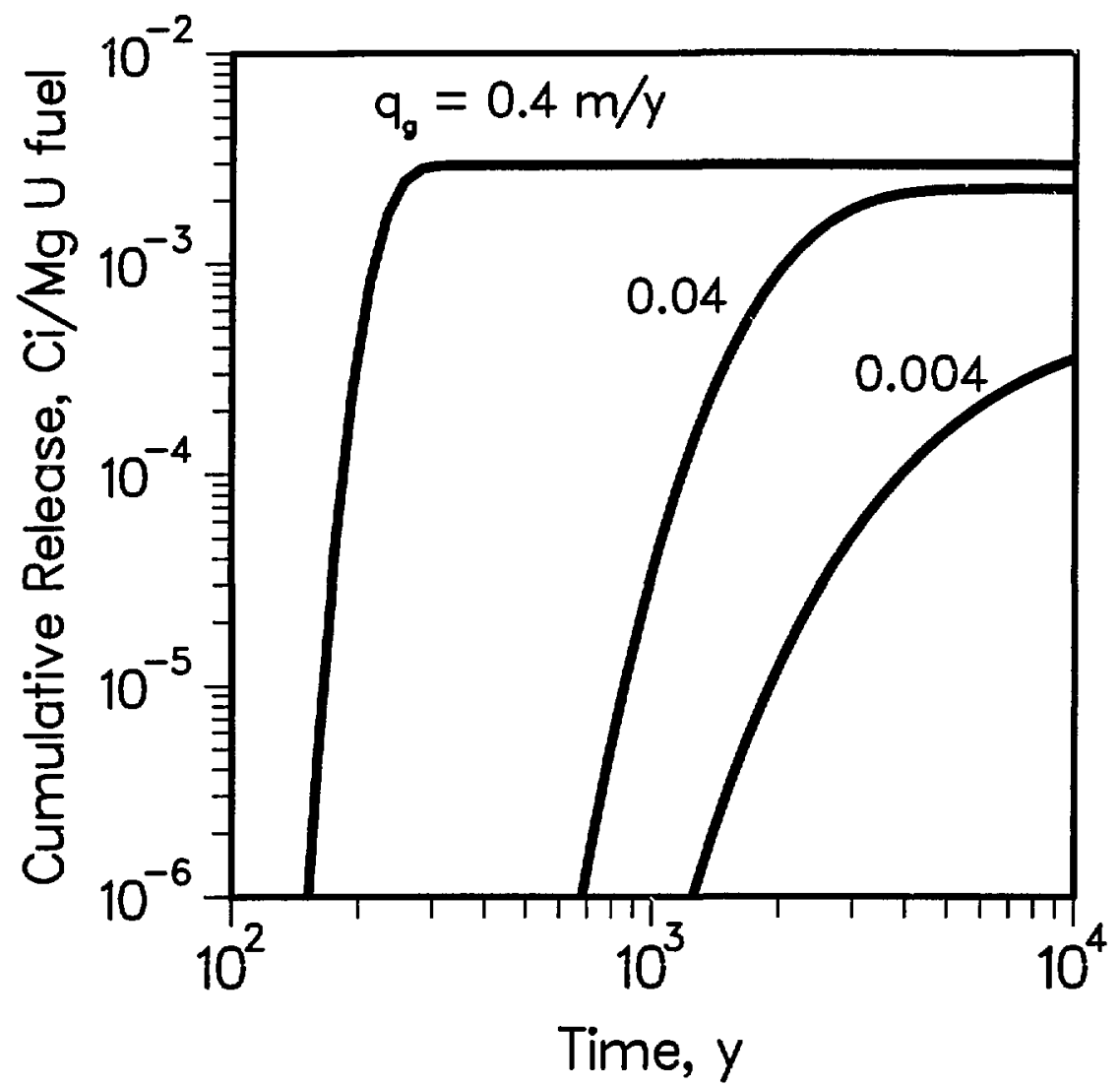

Figure 8. Cumulative release $350 \mathrm{~m}$ above infinite plane source for impulse release of $200 \mathrm{Ci}$ per $7 \times 10^{6} \mathrm{~m}^{2}(0.003 \mathrm{Ci} / \mathrm{Mg} \mathrm{U}$ fuel $)$. 


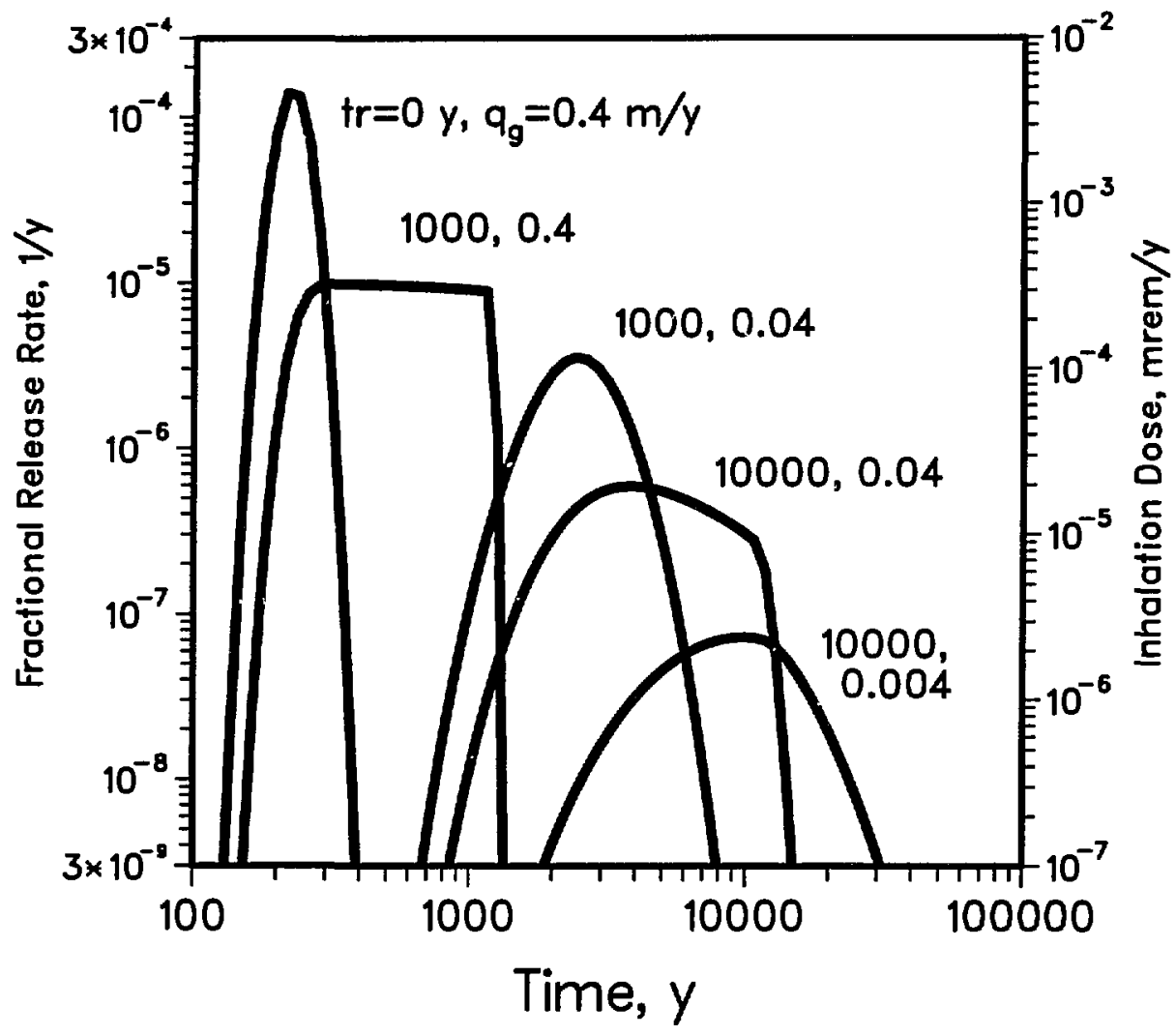

Figure 9. Inhalation dose and release rates $350 \mathrm{~m}$ above infinite plane source for various release durations and gas Darcy velocities. 
Table 1. Maximum dose above ground (mrem/y) as a function of gas Darcy velocity and band release duration for an infinite plane source with a releasable inventory of $1000 \mathrm{Ci}$ released as a decaying band in time over an area of $7 \times 10^{6} \mathrm{~m}^{2}, K_{D}=3$.

\begin{tabular}{|r|cccc|}
\hline $\begin{array}{c}\text { Release Duration } \\
\text { (years) }\end{array}$ & \multicolumn{4}{|c}{ Gas Darcy Velocity $(\mathrm{m} / \mathrm{y})$} \\
\hline 0 & $1 \times 10^{-2}$ & $1 \times 10^{-3}$ & $3 \times 10^{-5}$ & $1 \times 10^{-6}$ \\
300 & $2 \times 10^{-4}$ & $2 \times 10^{-4}$ & $3 \times 10^{-5}$ & $1 \times 10^{-6}$ \\
1000 & $7 \times 10^{-5}$ & $7 \times 10^{-5}$ & $2 \times 10^{-5}$ & $1 \times 10^{-6}$ \\
5000 & $1 \times 10^{-5}$ & $1 \times 10^{-5}$ & $8 \times 10^{-6}$ & $8 \times 10^{-7}$ \\
10000 & $7 \times 10^{-6}$ & $7 \times 10^{-6}$ & $4 \times 10^{-6}$ & $5 \times 10^{-7}$ \\
\hline
\end{tabular}

\section{CONCLUSION}

We have demonstrated a simple technique for estimating ${ }^{14} \mathrm{C}$ transport in the partially saturated, fractured medium at Yucca Mountain, requiring only a small set of well-defined parameter values.

Predicted gas concentrations approaching the ground surface are comparable to the unrestricted-area USNRC limit of $10^{-7} \mu \mathrm{Ci} / \mathrm{:m}^{3}$ in air. Dilution near the ground surface as the ${ }^{14} \mathrm{CO}_{2}$ enters the atmosphere will lower the concentration by several orders of magnitude. Predicted maximum dosages above ground are much lower than background radiation at sea level. For some gas velocities, the concentrations and fluxes at the ground surface are independent of the rate of release from the source and depend only on the total amount released. We estimate the travel time to the surface at Yucca Mountain to be hundreds to thousands of years, but caution that estimates are critically sensitive to the Darcy gas velocity and the distribution coefficient. If local equilibrium is not maintained, retardation may be less than predicted and concentration greater than predicted. For more definitive calculations the time- $c$ pendent gas velocities, temperatures, and local $\mathrm{pH}$ must be considered.

Solutions to porous medium equations are powerful but not always applicable to problems in fractured media. Our technique of determining suitability using the modif «d Peclet number will make it easier to defend the use of such solutions in many different systems.

\section{ACKNOWLEDGEMENT}

Work supported in part by the Director, Office of Civilian Radioactive Waste Management, Office of Systems Integration and Regulations, Licensing and Compliance Division, U. S. Department of Energy under contract DE-AC03-76SF00098. 


\section{NOMENCLATURE}

$a, b$ dummy variables

$A$ area of repository

$b$ half-width of fracture

$C$ source term constant $(16,20)$

$C_{g}$ molar concentration of ${ }^{14} \mathrm{C}$ in the gas phase

$C_{l}$ molar concentration of ${ }^{14} \mathrm{C}$ in the liquid phase

$D$ effective diffusion coefficient (38)

$\mathrm{D}_{g}, \mathrm{D}_{l}$ dispersion coefficient in gas,liquid phase

$D_{g}, D_{1}$ diffusion coefficient in gas,liquid phase with tortuosity

$f$ total suurce strength $f_{g}+f_{l}$

$f_{g}, f_{l}$ gas,liquid-jhase source term

$F$ function defined by (5i)

$g$ function defined by (52)

$G$ function defined by (46)

$h$ unit step function

$I_{0}$ inventory of ${ }^{14} \mathrm{C}$ released $(17,19,20)$

$I_{e}$ releasable inventory of ${ }^{14} \mathrm{C}$ at emplacement

$I_{f}$ releasable inventory of ${ }^{14} \mathrm{C}$ at failure

$K_{1}$ first dissociation equilibrium constant (3)

$K_{2}$ second dissociation equilibrium constant (3)

$K_{D}$ gas-liquid distribution coefficient $(1,2,3)$

$K_{H} \quad$ Henry's law constant (3)

$L$ lateral extent of repository (64)

$\dot{M}$ release rate at the source

$P$ modified Peclet number $(30,32)$

$\mathbf{q}_{g}, q_{g}$ gas Darcy velocity vector, scalar

$q_{l}, q_{l}$ liquid Darcy velocity vector, scalar

r position vector originating at point source

$S_{g l}$ net reaction rate from gas phase to liquid

$t, T$ time, dimensionless time (15)

$t_{f}$ time at failure

$t_{r}$ duration of release

$U$ mean wind speed (64)

$\checkmark$ effective advection velocity (38)

$v_{g}$ gas velocity in fracture

$y, Y$ horizontal distance, dimensionless horizontal distance

$z, Z$ vertical distance, dimensionless vertical distance

$\delta$ delta function

c total porosity

$\epsilon_{g}$ gas-filled porosity

$\epsilon_{l}$ liquid-filled porosity

$\phi$ vertical flux of ${ }^{14} \mathrm{C}$ (57)

$\lambda, \Lambda$ decay constant, dimensionless decay constant

$\sigma_{z}$ vertical mixing distance (64)

$\tau$ dummy variable

$\psi$ boundary condition source term $(7,16)$ 


\section{REFERENCES}

1. B. Ross, Gas-Phase Transport of Carbon-14 Released From Nuclear Waste Into the Unsaturated Zone, in Scientific Basis for Nuclear Waste Management $X I$, ed. M. J. Apted and R. E. Westerman (Mater. Res. Soc. Proc. 112, Pittsburgh, PA 1988) p. 23.

2. S. Amter, E. Behl and B. Ross, "Carbon-14 Travel Time at Yucca Mountain," Disposal Safety Incorporated, Washington, D.C., 1988.

3. R. B. Knapp, An Approximate Calculation of Advective Gas Phase Transport of ${ }^{14} \mathrm{C}$ at Yucca Mountain, Nevada, UCRL-97805, Lawrence Livermore Nationa! Laboratory, Livermore, C. A, 1987.

4. K. J. Schiager, Analysis of Radiation Exposures on or Near Uranium Mill Tailings Piles, Radiation Data and Reports, 15:7, 411 (1974).

5. W. B. Light, T. H. Pigford, P. L. Chambré and W. L.-L. Lee, ${ }^{14} \mathrm{C}$ Transport in a Partially Saturated, Fractured, Porous Medium, in Scientific Basis for Nuclear Waste Management XIII, ed. V. M. Oversby and P. W. Brown (Mater. Res. Soc. Proc. 176, Pittsburgh, PA 1990) p. 761.

6. W. B. Light, E. D. Zwahlen, T. H. Pigford, P. L. Chambré and W. W.-L. Lee, C-14 Release and Transport from a Nuclear Waste Repository in an Unsaturated Medium, $L B L-28929$, Lawrence Berkeley Laboratory, Berkeley, CA, 1990.

7. W. Stumm and J. J. Morgan, Aquatic Chemistry, 2nd Ed. (John Wiley \& Sons, New York, 198I).

8. S. L. Phillips, C. A. Phillips and J. Sieen, Hydrolysis, Formation and Ionization Constants at $25^{\circ} \mathrm{C}$, and at High Temperatures - High Ionic Strength, $L B L-14996$, Lavirence Berkeley Laboratory, Berkeley, CA, 1985 .

9. L. E. Berman, D. A. Ensminger, M. S. Giufre, C. M. Koplik, S. G. Osion, G. D. Pollak, B. I. Ross and A. A. Sutherland, Analysis of Some Nuclear Waste Management Options, Volume II: Appendices, UCRL-19917, 1978.

10. K. H. Haskell and R. E. Jones, Brief Instructions for Using the Sandia Mathematical Subroutine Librar\} (Version 7.2), SAND77-1441, Sandia National Labcratories, Albuquerque, NM, 1978.

11. National Council on Radiation Protection and Measurements, "Screening Techniques for Determining Compliance with Environmental Standards: Releases of Radionuclides to the Atmosphere," NCRP Commentary 9, 1989.

12. Y. W. Tsang and K. Pruess, A Study of Thermally Induced Convection Near a High-Level Nuclear Waste Repository in Partially Saturated Fractured Tuff, Water Resourcfs Res. 29, 1958 (1987).

13. R. T. Green and D. D. Evans, Radionuclide Transport as Vapor Through Unsaturated Fractured Rock, NUREG/CR-4654, University of Arizona, Tucson, AZ, 1987.

14. R. R. Heters, E. A. Klavetter, I. J. Hall, S. C. Blair, P. R. Heller, and G. W. Gee, Fracture and matrix hydrologic characteristics of tuffaceous materials from Yucca Mountain, Nye County, Nevada, SAND841/71, Sandia National Laboratories, Albuquerque, NM, 1984.

15. U.S. Department of Energy, Site Characterization Plan, Yucca Mountain Site, DOE/RW-0199, 8.3.5.14$4,1988$. 
Appendix A. Derivation of Discrete-Fracture Solution

In dimensionless variables the equation system (5)-(12) becomes

$$
\begin{aligned}
\frac{\partial C_{s}}{\partial T}+\Lambda C_{g}+\frac{\partial C_{g}}{\partial Z}=\left.\frac{1}{K_{D}} \frac{\partial C_{l}}{\partial Y}\right|_{Y=0} \quad Z>0 \quad T>0 \\
\frac{\partial C_{l}}{\partial T}+\Lambda C_{l}-\frac{\partial^{2} C_{l}}{\partial Y^{2}}=0 \quad Y>0 \quad Z>0 \quad T>0 \\
C_{g}(Z, 0)=0 \quad Z>0 \\
C_{l}(Y, Z, 0)=0 \quad Y>0 \quad Z>0 \\
C_{s}(0, T)=\psi(T) \quad T>0 \\
C_{g}(\infty, T)=0 \quad T>0 \\
C_{l}(0, Z, T)=K_{D} C_{g}(Z, T) \quad Z>0 \quad T>0 \\
C_{1}(\infty, Z, T)=0 \quad Z>0 \quad T>0
\end{aligned}
$$

The solution is obtained using Laplace transforms. First (A.1) and (A.2) are transformed with respeci to $T$ using the initial conditions (A.3) and (A.4)

$$
\begin{gathered}
\frac{\partial \tilde{C}_{g}}{\partial Z}+(p+\Lambda) \tilde{C}_{g}=\left.\frac{1}{K_{D}} \frac{\partial \tilde{C}_{l}}{\partial Y}\right|_{Y=0} \quad Z>0 \\
\frac{\partial^{2} \tilde{C}_{l}}{\partial Y^{2}}-(p+\Lambda) \tilde{C}_{l}=0 \quad Y>0 \quad Z>0
\end{gathered}
$$

The solution to the ordinary differential equation (A.10), using boundary conditions (A.7) and (A.8) is

$$
\tilde{C}_{l}=K_{D} \tilde{C}_{g} \exp [-Y \sqrt{p+\Lambda}] \quad Y>0 \quad Z>0
$$

Substituting (A.11) into (A.9) gives

$$
\frac{\partial \tilde{C}_{g}}{\partial Z}+(p+\Lambda) \tilde{C}_{y}=-\sqrt{p+\Lambda} \tilde{C}_{g} \quad Z>0
$$

We need boundary conditions (A.5) and (A.6) to solve this equation with the result

$$
\tilde{C}_{g}=\tilde{\psi} \exp [-Z(p+\Lambda+\sqrt{p+\Lambda})] \quad Z>0
$$

To get the inverse Laplace transform of (A.13) we break it into factors and apply the convolution theoren. First let

$$
\tilde{u}_{1}(Z, p)=\exp [-Z(p+\Lambda+\sqrt{p+\Lambda})] \quad Z>0
$$

The inverse of (A.13) can then be written as the convolution integral

$$
C_{g}(Z, T)=\int_{\zeta}^{T} \psi(T-\tau) u_{1}(Z, \tau) d \tau
$$

To get $u_{1}(Z, T)$ use the p-shift property to get

$$
u_{1}(Z, T)=e^{-\Lambda T} u_{2}(Z, T)
$$


where

$$
\bar{u}_{2}(Z, p)=\exp [-Z(p+\sqrt{p})]
$$

This in turn is redisced using the $T$-shift property to

$$
u_{2}(Z, T)=h(T-Z) u_{3}(T-Z)
$$

where

$$
\bar{u}_{3}(Z, p)=\exp [-Z \sqrt{p}]
$$

The inverse Laplace transform of (A.19) is

$$
u_{3}(Z, T)=\frac{Z}{2 \sqrt{\pi T^{3}}} \exp \left(\frac{-Z^{2}}{4 T}\right)
$$

Putting the last several equations together gives us

$$
C_{g}(Z, T)=\int_{0}^{T} \psi(T-\tau) e^{-\Lambda \tau} h(\tau-Z) \frac{Z}{2 \sqrt{\pi(\tau-Z)^{3}}} \exp \left(\frac{-Z^{2}}{4(\tau-Z)}\right) d \tau \quad Z>0 \quad T>0
$$

or, after rearranging

$$
C_{g}(Z, T)-e^{-\Lambda Z} h(T-Z) \int_{0}^{T-Z} \psi(T-Z-\tau) \frac{Z}{2 \sqrt{\pi \tau^{3}}} \exp \left(\frac{-Z^{2}}{4 \tau}-\Lambda \tau\right) d r \quad Z>0 \quad T>0
$$

Substituting $\tilde{C}_{g}$ from (A.13) into (A.11) gives

$$
\tilde{C}_{l}(Y, Z, p)=K_{D} \tilde{\psi} \exp [-Z(p+\Lambda)] \exp [-(Z+Y) \sqrt{p+\Lambda}] \quad Y>0 \quad Z>0
$$

To invert this we use the same method as before obtaining first the convolution integral

$$
C_{l}(Y, Z, T)=K_{D} \int_{0}^{T} \psi(T-\tau) u_{1}(Y, Z, \tau) d \tau
$$

where

then by the p-shift property

$$
\tilde{u}_{1}(Y, Z, p)=\exp [-Z(p+\Lambda)] \exp [-(Z+Y) \sqrt{p+\Lambda}]
$$

$$
u_{1}(Y, Z, T)=e^{-\Lambda T} u_{2}(Y, Z, T)
$$

where

then by the $T$-shift property

$$
\tilde{u}_{2}(Y, Z, p)=\exp (-Z p) \exp (-(Z+Y) \sqrt{p})
$$

where

$$
u_{2}(Y, Z, T)=h(T-Z) u_{3}(\Gamma-Z)
$$

$$
\tilde{u}_{3}(Y, Z, p)=\exp (-(Z+Y) \sqrt{p})
$$

This is inverted as before to give

Putting it all together gives the result

$$
u_{3}(Y, Z, T)=\frac{Z+Y}{2 \sqrt{\pi T^{3}}} \exp \left(\frac{-(Z+Y)^{2}}{4 T}\right)
$$

$C_{i}(Y, Z, T)=K_{D} \int_{0}^{T} \psi(T-\tau) e^{-\Lambda \tau} h(\tau-Z) \frac{Z+Y}{2 \sqrt{\pi(\tau-Z)^{3}}} \exp \left(\frac{-(Z+Y)^{2}}{4(\tau-Z)}\right) d \tau \quad Y>0 \quad Z>0 \quad T>0$

or alternatively

$C_{l}(Y, Z, T)=K_{D} e^{-\Lambda z} h(T-Z) \int_{0}^{T-Z} \downarrow(T-Z-\tau) \frac{Z+Y}{2 \sqrt{\pi \tau^{3}}} \exp \left[\frac{-(Z+Y)^{2}}{4 \tau}-\Lambda \tau\right] d \tau \quad Y>0 \quad Z>0 \quad T>0$ 
Appendix B. Cubic Polynomi ; Root Finding

The following polynomial theory is from CRC Standard Mathematical Tables, 27th Edition, by William H. Beyer, CRC Press, Inc., Boca Raton, Florida, 1984.

Any cubic polonomial

$$
y^{3}+p y^{2}+q y+r=0
$$

can be transformed by setting $y=x-p / 3$ to

$$
x^{3}+a x+b=0
$$

with

$$
a=\frac{1}{3}\left(3 q-p^{2}\right) \quad b=\frac{1}{27}\left(2 p^{3}-9 p q+27 r\right)
$$

For $p, q, r$ real then

$$
\begin{aligned}
& \frac{b^{2}}{4}+\frac{a^{3}}{27}>0 \rightarrow 1 \text { real, } 2 \text { conjugate complex roots } \\
& \frac{b^{2}}{4}+\frac{a^{3}}{27}=0 \rightarrow 3 \text { real roots, at least } 2 \text { equal } \\
& \frac{b^{2}}{4}+\frac{a^{3}}{27}<0 \rightarrow 3 \text { real, unequal roots }
\end{aligned}
$$

For $a b \neq 0$ the roots can be obtained by letting $x=m \cos \theta$ to get

$$
\begin{aligned}
x^{3}+a x+b & =m^{3} \cos ^{3} \theta+a m \cos \theta+b \\
& =4 \cos ^{3} \theta-3 \cos \theta-\cos (3 \theta)=0
\end{aligned}
$$

Hence

$$
\frac{4}{m^{3}}=\frac{-3}{a m}=\frac{-\cos (3 \theta)}{b}
$$

or

$$
m=2 \sqrt{\frac{-a}{3}} \quad \cos (3 \theta)=\frac{3 b}{a m}
$$

The roots aie

$$
x=2 \sqrt{\frac{-a}{3}} \cos (\theta) \quad 2 \sqrt{\frac{-a}{3}} \cos \left(\theta+\frac{2 \pi}{3}\right) \quad 2 \sqrt{\frac{-a}{3}} \cos \left(\theta+\frac{4 \pi}{3}\right)
$$

where $\theta$ is a solution to (B.6).

We have

$$
p=-2(1+T) \quad q=-2 T \quad r=4 T^{2}
$$

which leads to

$$
a=\frac{-2}{3}\left(2 T^{2}+7 T+2\right) \quad b=\frac{-4}{27}\left(4 T^{3}-6 T^{2}+21 T+4\right)
$$

It can then be determined by substitution and much pencil work that $\frac{b^{2}}{4}+\frac{a^{3}}{27}<0$ for $T$ positive leading to the conclusion that our original cubic polynomial has three distinct, real roots. Going back to (B.6) we have

$$
m=2 \sqrt{\frac{-a}{3}}=\frac{2}{3} \sqrt{4 T^{2}+14 T+4}
$$

and

$$
\cos (3 \theta)=\frac{3 b}{a m}=\left[\frac{8 T^{3}-12 T^{2}+42 T+8}{\left(4 T^{2}+14 T+4\right)^{3 / 2}}\right]
$$


We can now look at the early time limit to see which root gives us $Z_{\text {peak. }}$. The early time limit for $m$ in $(\mathrm{B} .10)$ is

$$
\lim _{T \rightarrow 0} m=\frac{4}{3}
$$

and for $\cos (3 \theta)$ in $(B .11)$

$$
\lim _{T \rightarrow 0} \cos (3 \theta)=1
$$

Solutions to this equation are $\theta=0, \frac{2 \pi}{3}, \frac{4 \pi}{3}$, etc. The early-time limits of the roots to the transformed polynomial $x=m \cos \theta$ are then $x=\frac{4}{3}, \frac{-2}{3}, \frac{-2}{3}$ and from this the roots to the original polynomial in the early-time limit

$$
\lim _{T \rightarrow 0} Z=\lim _{T \rightarrow 0}(x-p / 3)=x+2 / 3=2,0,0
$$

This narrows our choice for $Z_{\text {peak }}$ to the second or third root corresponding to $\theta=\frac{2 \pi}{3}$ or $\frac{4 \pi}{3}$. We can write the root in terms of an arccos function but we do not know yet which branch of this multivalued function to take

$$
Z=\frac{2}{3} \sqrt{4 T^{2}+14 T+4} \cos \left[\frac{1}{3} \arccos \left(\frac{8 T^{3}-12 T^{2}+42 T+8}{\left(4 T^{2}+14 T+4\right)^{3 / 2}}\right)\right]+\frac{2}{3}(1+T)
$$

By trial and error we determine that $\left(\frac{1}{3} \operatorname{Arccos}()+\frac{4 \pi}{3}\right)$ is the correct branch to use so we have

$$
Z_{\text {peak }}=\frac{2}{3}(T+1)+\frac{2}{3} \sqrt{4 T^{2}+14 T+4} \cos \left(\frac{1}{3} A \operatorname{rccos}\left[\frac{8 T^{3}-12 T^{2}+42 T+8}{\left(4 T^{2}+14 T+4\right)^{3 / 2}}\right]+\frac{4 \pi}{3}\right)
$$

The following alternate form is better suited for numerical evaluation when $T$ is greater than one.

$$
Z_{\text {peak }}=\frac{2}{3}(T+1)\left[1+\frac{\sqrt{4+14 / T+4 / T^{2}}}{1+1 / T} \cdot \cos \left(\frac{1}{3} \operatorname{Arccos}\left[\frac{8-12 / T+42 / T^{2}+8 / T^{3}}{\left(4+14 / T+4 / T^{2}\right)^{3 / 2}}\right]+\frac{4 \pi}{3}\right)\right]
$$




\section{Appendis c. Derivation of Equivalent Porous Medium Solutions}

Solutions to (37) for transport of a radionuclide in a porous medium with advection, diffusion and retardation can be obtained with the use of Kelvin's function for various source terms, boundary conditions and initial conditions. This method follows the more general treatment given by P. L. Chambré et al. in Chapter 10 of "Mass Transfer and Trainsport in a Geologic Environment", LBL-19430, Lawrence Berkeley Laboratory, University of California, 1985.

We restrict ourselves to solutions of an infinite domain with a concentration of zero initially and zero at infinity for all time. Solutions will be given for a point source and an infinite plane source with a constant velocity $\mathbf{v}$ in the $z$ direction. Mathematical expressions for the source terms are given by (39)-(43).

To remove the decay term, the dependent variable $C_{g}$ is replaced by

$$
c(r, t)=e^{\lambda t} C_{g}(r, t)
$$

to yield

$$
\frac{\partial}{\partial t} \mathbf{c}(\mathbf{r}, t)+\mathbf{v} \cdot \nabla c(\mathbf{r}, t)-D \nabla^{2} c(\mathbf{r}, t)=\frac{e^{\lambda t} f(\mathbf{r}, t)}{\epsilon_{g}+\epsilon_{l} K_{D}}
$$

or, in cartesian space coordinates with $\mathrm{v}$ in the $z$ direction

$$
\frac{\partial c}{\partial t}+v \frac{\partial c}{\partial z}-D\left(\frac{\partial^{2} c}{\partial x^{2}}+\frac{\partial^{2} c}{\partial y^{2}}+\frac{\partial^{2} c}{\partial z^{2}}\right)=\frac{e^{\lambda t} f(x, y, z, t)}{\epsilon_{g}+c_{3} K_{D}}
$$

For a point source term (39) the right hand side of (C.3) is homogeneous away from the source point. Solutions can be written in the product form

$$
\mathfrak{c}(x, y, z, t)=c_{1}(x, t) c_{2}(y, t) c_{3}(z, t)
$$

where $c_{1}, c_{2}$ and $c_{3}$ satisfy respectively

$$
\begin{gathered}
\frac{\partial c_{1}}{\partial t}-D \frac{\partial^{2} c_{1}}{\partial x^{2}}=0 \\
\frac{\partial c_{2}}{\partial t}-D \frac{\partial^{2} c_{2}}{\partial y^{2}}=0 \\
\frac{\partial c_{3}}{\partial t}+v \frac{\partial c_{3}}{\partial z}-D \frac{\partial^{2} c_{3}}{\partial z^{2}}=0
\end{gathered}
$$

To remove the advection term in (C.7) the dependent variable $z$ is replace by

$$
\zeta=z-v t
$$

with

$$
c_{3}(z, t)=C_{3}(\zeta, t)
$$

yielding

$$
\frac{\partial C_{3}}{\partial t}-D \frac{\partial^{2} C_{3}}{\partial \zeta^{2}}=0
$$

The Green's function for (C.10) is the solution to

$$
\frac{\partial G}{\partial t}-D \frac{\partial^{2} G}{\partial \zeta^{2}}=\delta(\zeta) \delta(t)
$$

with a one-dimensional point source singularity of unit strength at $\zeta=0, t=0$ and is given by the well known Kelvin function

$$
G(\zeta, t)=\frac{1}{\sqrt{4 \pi D t}} \exp \left\{\frac{-\zeta^{2}}{4 D t}\right\}
$$


Thus the Green's functions for (C.5) and (C.6) are similarly

$$
g_{1}(x, t)=\frac{1}{\sqrt{4 \pi D t}} \exp \left\{\frac{-x^{2}}{4 D t}\right\}
$$

and

$$
g_{2}(y, t)=\frac{1}{\sqrt{4 \pi D t}} \exp \left\{\frac{-y^{2}}{4 D t}\right\}
$$

and the Green's function for (C.7) from (C.12) is

$$
g_{3}(z, t)=\frac{1}{\sqrt{4 \pi D t}} \exp \left\{\frac{-(z-v t)^{2}}{4 D t}\right\}
$$

The Green's function for (C.3) is the solution to

$$
\frac{\partial g}{\partial t}+v \frac{\partial g}{\partial z}-D\left(\frac{\partial^{2} g}{\partial x^{2}}+\frac{\partial^{2} g}{\partial y^{2}}+\frac{\partial^{2} g}{\partial z^{2}}\right)=\delta(x) \delta(y) \delta(z) \delta(t)
$$

with a three-dimensional point source singularity of unit strength at the origin and is given by the product of the Green's functions for (C.5)-(C.7)

$$
g(x, y, z, t)=\frac{1}{(4 \pi D t)^{3 / 2}} \exp \left\{\frac{-\left(x^{2}+y^{2}+(z-v t)^{2}\right)}{4 D t}\right\}
$$

or, in vector notation

$$
g(\mathbf{r}, t)=\frac{1}{(4 \pi D t)^{3 / 2}} \exp \left\{\frac{-(\mathbf{r}-\mathbf{v} t)^{2}}{4 D t}\right\}
$$

In turn, the solution due to a point source at the origin, releasing the amount $\dot{M}(\tau) d \tau$ at time $\tau$ during the time span $d \tau$, creates the contribution at time $t$

$$
d c=\left(\frac{e^{\lambda \tau} \dot{M}(\tau)}{\epsilon_{g}+c_{1} K_{D}} d \tau\right) g(x, y, z, t-\tau) \quad t>\tau
$$

Hence the total contribution due to the amount released during the time span $0^{-}<\tau<t$ is given by the superposition integral

$$
c(x, y, z, t)=\int_{0^{-}}^{t}\left(\frac{e^{\lambda r} \dot{M}(\tau)}{\epsilon_{g}+\epsilon_{l} K_{D}}\right) g(x, y, z, t-\tau) d \tau
$$

Substitution for $c$ (C.1) and $g$ (C.18) leads directly to the solution (44) for a point source with a time dependent release rate.

In the case of an infinite plane source, (C.3) reduces to

$$
\frac{\partial c}{\partial t}+v \frac{\partial c}{\partial z}-D \frac{\partial^{2} c}{\partial z^{2}}=\frac{e^{\lambda t} f(z, t)}{\epsilon_{g}+\epsilon_{l} K_{D}}
$$

The solution due to an infinite plane source at the origin, releasing the amount per unit area $(\dot{M}(\tau) / A) d \tau$ at time $\tau$ during the time span $d \tau$, creates the contribution at time $t$

$$
d c=\left(\frac{e^{\lambda \tau}(\dot{M}(\tau) / A)}{\epsilon_{g}+\epsilon_{l} K_{D}} d \tau\right) g_{3}(z, t-\tau) \quad t>\tau
$$

The total contribution due to the amount released during the time span $0^{-}<\tau<t$ is given by the superposition integral

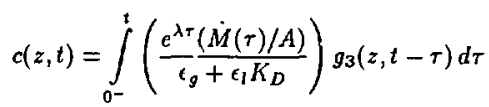

Substitution for $c$ (C.1) and $g_{3}$ (C.15) lead to vhe solution (45) for the infinite plane source with time dependent release rate. 